\title{
A DISCRETE VARIATIONAL APPROACH FOR INVESTIGATION OF STATIONARY LOCALIZED STATES IN A DISCRETE NONLINEAR SCHRÖDINGER EQUATION, NAMED IN-DNLS
}

\author{
K. KUNDU
}

Received 9 June 2004

IN-DNLS considered here is a countable infinite set of coupled one-dimensional nonlinear ordinary differential difference equations with a tunable nonintegrability parameter. When this parameter vanishes, IN-DNLS reduces to the famous integrable AblowitzLadik (AL) equation. The formation of unstaggered and staggered stationary localized states (SLSs) in IN-DNLS is studied here using a discrete variational method. The functional form of stationary soliton of AL equation is used as the ansatz for SLSs. Derivation of the appropriate functional and its equivalence to the effective Lagrangian are presented. Formation of on-site peaked and intersite peaked unstaggered SLSs and their dependence on the nonintegrability parameter are investigated. On-site peaked states are found to be energetically stable. Results are explained using the effective mass picture. Also, the properties of staggered SLSs of Sievers-Takeno- (ST-) like mode and Page- (P-) like mode are investigated and explained using the same effective mass picture. It is further shown here that an unstable SLS which is found in the truncated analysis of the problem does not survive in the exact calculation. For large-width and small-amplitude SLSs, the known asymptotic result for the amplitude is obtained. Further scope and possible extensions of this work are discussed.

\section{Introduction}

The study of energy localization in nonlinear lattices has become an important field of research in nonlinear dynamics in the past couple of decades [25]. In this context, the subject of intrinsic localized modes (ILMs) has drawn a considerable attention as it offers appealing insights into a variety of problems ranging from the nonexponential energy relaxation [69] in solids to the local denaturation of DNA double strands [14, 59]. The subject is also an intense field of study in material science, and nonlinear optic applications $[23,49,66]$.

The necessary condition for the formation of intrinsic localized modes (ILMs) or excitations in translationally invariant nonlinear systems is the balance between nonlinearity and dispersion. Furthermore, by localized it is meant that the amplitude of such modes 
goes to zero at the boundaries of the system, which is taken to be infinitely large. In other words, the relevant localization length scale is much smaller than the system size length scale. There are two broad classes of intrinsic localizations in $(1+1)$-dimensional nonlinear continuous systems [68]. Shape preserving localized excitations, arising in nonlinear continuous systems by satisfying the above-mentioned balancing condition, are called dynamical solitons $[20,61,64]$. Solitons in continuous nonlinear Schrödinger equation (CNLS) is an example of dynamical solitons $[61,71]$. By solitons we usually mean moving shape preserving nonlinear excitations, though there can be stationary solitons also. Take for example CNLS. One particular one-soliton solution of this equation is a stationary soliton [71]. Breathers belong to the second category of ILMs in nonlinear systems [68]. Breathers are spatially localized time-periodic solutions of nonlinear equations. They are characterized by internal oscillations $[7,8,9,10,11,20,24,26,40,44,50,54,55,60,68]$. Again, by breathers we usually imply stationary localized excitations in nonlinear systems. However, under appropriate conditions, nonlinear systems may have moving breathers [20]. As for examples, we note that breathers can be found in continuous systems, described by sine-Gordon (SG) equation and modified KdV (mKdV) equation [20]. Even in CNLS, the stationary one-soliton solution is nothing but a breather [26]. So, the distinction between solitons and breathers is not always very rigorous. Breathers are however rare objects in continuous nonlinear equations and are usually unstable [26]. It is important in the present context to note that continuous nonlinear equations may have Galilean or Lorentz invariance. For example, KdV and CNLS are Galilean invariant [20]. So, a soliton of some fixed amplitude in the CNLS and KdV can be Galileo boosted to any velocity. Similarly, an SG equation has both stationary and moving breather solutions $[9,20]$. These two solutions are, however, connected by Lorentz transformation [20]. So, in dealing with stationary ILMs, we consider that moving frame which is at rest with respect to the ILM.

However, models describing a microscopic phenomenon in condensed matter physics are inherently discrete, with the lattice spacing between atomic sites being a fundamental physical parameter. For these systems, an accurate microscopic description involves a set of coupled ordinary differential difference equations (ODDEs). Coupled ODDEs are also encountered in the study of many important problems in optics and other branches of science $[5,9,26]$. So, it is pertinent to discuss next what features of continuous nonlinear equations are possibly destroyed and what novel features can arise from the discretization of at least one of the variables, say one spatial dimension.

In the general discrete case, Galilean or Lorentz invariance in relevant dynamical equations may not be present at all or may not be transparent at the equation level. Consider, for example, the AL $[1,2,3]$ and the N-AL equations [46]. The first one is the example of an integrable nonlinear differential discrete equation, which is often referred to as the integrable discretization of the CNLS equation. The other equation provides an example of a differential discrete nonintegrable nonlinear equation, having solitary wave solutions. Most importantly, the existence of solitary waves in the N-AL equation can be shown analytically [46]. The solitary wave solutions of these equations have continuous translational symmetry, which can be seen from the analytical expression of the onesoliton solution of the AL equation. This in turn implies that both the AL and the N-AL 
equations have the Galilean invariance. So, also in case of ODDEs, stationarity in the ILM will imply the moving frame which is at rest with respect to the ILM.

The replacement of the spatial derivatives by spatial differences in the equation of motion implies the reduction of symmetry of the Hamiltonian, for systems executing Hamiltonian dynamics. In general, lowering the symmetry means enriching the class of solutions, because less restrictions are imposed. Of course, solutions are also lost by lowering the symmetry, namely, ones which are generated by higher symmetry [26]. We consider in this context two discrete nonlinear equations, the Frenkel-Kontorova (FK) $[9,15]$ and the discrete nonlinear Schrödinger (DNLS) equations [45]. These are obtained by standard discretization of SG and CNLS, respectively $[5,9,19,45]$. The FK model can be used to describe a broad spectrum of physically important nonlinear phenomena, such as propagation of charge-density waves, the dynamics of absorbed layer of atoms on crystal surfaces, commensurable-incommensurable phase transitions, domain walls in magnetically ordered structures and so forth $[9,15]$. On the other hand, to name a few, DNLS has been used to model the self-trapping phenomenon in nonlinear waveguide arrays [5], to investigate a slow coherent transport of polarons in $(1+1)$ dimension in condensed matter physics [45], and to study the dynamical phase diagram of dilute Bose-Einstein condensates [68]. We note that both of these discrete equations are nonintegrable while their continuous versions are integrable. It is relevant in this context to know that kink and antikink solutions of SG equation, which is the continuous integrable version of FK model are moving topological solitons, and they arise due to the balance between nonlinearity and constraints originating from topological invariants in the system [61]. On the other hand, there exists no steady-state solutions for a moving kink in the FK model. What we obtain instead is static kinks [9]. To understand this, we note that the uniform discretization of space variables transforms continuous translational invariance to lattice translational invariance. This in turn leads to a periodic arrangement of Peierls-Nabarro (PN) potential $[9,41]$. Therefore, while the continuous translational invariance leads to zero frequency Goldstone modes in the system, discreteness introduces the PN barrier, with the barrier energy $E_{\mathrm{PN}}$ [9]. Due to this potential, any moving kink radiates phonons and loses energy $\left(E_{\text {kink }}\right)$. When $E_{\text {kink }}<E_{\mathrm{PN}}$, the kink is trapped in one of the potential wells and further loss of energy by the kink by radiation of phonons takes it to the bottom of the well. This in turn yields static kinks. Similarly, SG breathers arise due to the high symmetry of the equation and consequently are unstable towards perturbation [26]. As the discreteness in space variables act as an external symmetry breaking perturbation, even a weak discreteness does not allow oscillating breather modes to exist as dynamical eigenmodes of the SG chain, and breathers are destroyed by radiation of linear waves. In case of DNLS, similar analysis has been done in a perturbative frame using AL onesoliton solution as the zeroth order approximation $[45,70]$. This analysis also shows that discreteness introduces a trapping potential for moving solitons and when discreteness exceeds a critical value, solitonic modes are trapped leading ultimately to pinned or stationary solitons.

It is already mentioned that the AL equation is an integrable discrete nonlinear equation. More specifically, the said equation is a countably infinite set of one-dimensional nonlinear ordinary differential difference equations. This equation is continuous in time, 
but discrete in space with lattice translational invariance. The exact one-soliton solution of the AL equation is characterized by two parameters, namely, $\beta \in[0, \infty)$ and $k \in[-\pi, \pi]$ $[1,2,3]$. For each $\beta$, there exists a band of velocities determined by the other parameter $k$, at which the soliton can travel without experiencing any PN pinning from the lattice discreteness [13]. Consider now other nonlinear equations in this series, namely, the NAL equation [46], the modified Salerno equation (MSE) [45, 62], and the IN-DNLS [13]. All these equations are nonintegrable extension of the AL equation, containing tunable nonlinearities. The N-AL equation is postulated and investigated to study the effect of dispersive imbalance on the maintenance of the moving solitonic profile. The importance of this equation lies in its appearance in the dynamics of vibrons and excitons in soft molecular chains $[45,46]$. The solitary wave solutions of this equation are also characterized by the same two AL parameters, $\beta$ and $k$. However, only certain values of $k$ are allowed, though $\beta$ can take all possible permissible values. At the allowed values of $k$, the term which imparts nonintegrability disappears. This in turn makes the solitary waves transparent to the PN potential, arising from the lattice discreteness. For other values of $k$, the initial AL one-soliton profiles are observed numerically to leave phonon tails behind, causing both slowing down and distortion of the initial profile. Important too in this context is an analytical investigation in a perturbative framework of the dynamics of a moving AL soliton, described by the N-AL equation. This analysis suggests that any moving soliton having energy below the PN barrier, induced by the discreteness in the lattice will be pinned, yielding thereby stationary solitons [46].

The IN-DNLS is a hybrid form of the AL equation and the DNLS, again with a tunable nonlinearity, the tuning of which switches the equation from the integrable AL equation to the nonintegrable DNLS [13]. To gauge the physical significance of this equation, we mention the following. This equation is studied to investigate the discreteness-induced oscillatory instabilities of dark solitons [37, 42]. Furthermore, a discrete electrical lattice where the dynamics of modulated waves can be modeled by this equation is studied to investigate the modulation instability of plane waves [56]. In the MSE, the usual DNLS is replaced by a modified version of DNLS, the ADNLS, which involves acoustic phonons instead of optical phonons in condensed matter physics parlance $[45,70]$. The study of this equation is also important in understanding the dynamics of vibrons and excitons in soft molecular chains. It is important to note that both IN-DNLS and MSE investigate the competition between the on-site trapping and the solitonic motion of the AL soliton $[12,13,45]$. So, the dynamics of a moving self-localized pulse, like the AL soliton in the framework of the IN-DNLS or the MSE, will be subjected to two important effects. The first one is the PN pinning arising from the lattice discreteness and the second one is a nonlinear interaction potential trying to trap or detrap the localized pulse. The cumulative effect of these two interactions is expected to be the collapse of the moving self-localized states to stable, but pinned solitons. This has indeed been observed in a numerical simulation [13]. From this discussion so far, it can be concluded that the sufficient condition to see the effect of discreteness on the dynamics of nonlinear excitations is that the discrete nonlinear equations must be nonintegrable. This nonintegrability can arise directly from the discretization of the continuous nonlinear equations or by adding integrability breaking terms to integrable discrete nonlinear equations. 
Two important linear PDEs, which play very important roles in physics in linear systems are free-particle Schrödinger equation and the wave equation, respectively [22]. These equations are of course used to describe dynamics in continuous systems. The eigenvalue spectra of these equations are a continuous function of a parameter $k$, called wave vector, with the $\overline{\lim }=\infty$ and the $\underline{\lim }=0$. In case of systems, described by Schrödinger equation with a single-particle potential, an attractive potential will create localized states below the spectra and these are called "bound states" of the system [22]. Furthermore, in one-( $1+1)$-dimensional systems, even an infinitesimally small attractive potential will create an exponentially localized bound state. On the other hand, when wave equation is second order in time, even in $(1+1)$ dimension, no attractive potential, however large, can create bound states. On the contrary, one can get resonances from attractive potentials.

When the continuity in spatial variables is replaced by lattice continuity, the continuous spectra of linear PDEs fragment into bands. The number of bands will depend on the number of lattice points in the unit cell. When linear substitutional impurities are added to systems, described by a discrete Schrödinger equation, spatially localized states are formed in the gap between bands [22, 36, 57]. We note that for a state to be localized and stable, it must be in the gap of the spectra. Furthermore, these states, being exact eigenstates of the relevant Hamiltonian, are stationary localized states (SLSs). For a finite number of linear impurities in $(1+1)$ dimension, it can be shown that the number of spatially exponentially localized states cannot exceed the number of impurities and there must be at least one exponentially localized state [22]. On the other hand, almost all states are exponentially localized in fully disordered $(1+1)$-dimensional systems $[36,57]$. However, with correlated disorder, it is possible to have some delocalized states [21, 47]. Instead of linear impurities, if a finite number of nonlinear impurities are present, we again obtain SLSs in such systems. This can be analytically shown in the systems described by the DNLS [27, 29, 30, 31, 32, 48].

The spatially discrete analog of the continuous wave equation is the coupled massspring systems, with springs obeying Hooke's law [22, 36, 57]. Here again we get bands of eigenmodes, depending on the number of mass-spring units in a unit cell. The lowest band is called an acoustic branch, which describes the collective motion of the masses. Other bands give optical phonons [72]. In systems containing a finite number of mass impurities, only light mass impurities will form exponentially localized states above the acoustic band in $(1+1)$ dimension. A similar result is also obtained with impurity in springs [22]. Here also almost all states are exponentially localized in totally disordered systems, whether the disorder is in the mass or in the spring or in both [36, 57]. However, no states are obtained below the acoustic branch. Most importantly, states around zero frequency remain delocalized $[36,57]$. In this system also, one can have nonlinear impurities, in the spring, in the on-site potential, or in both. Any such impurity will produce SLSs in the system [43]. We end this discussion by noting that both continuous and discrete linear systems cannot sustain any localized mode without broken continuous and lattice translational invariance, respectively.

A uniform discrete nonlinear system will have lattice translational invariance. Similarly to continuous nonlinear systems with translational invariance, nonlinearity in discrete 
systems can also generate localized modes by balancing the delocalization effect without requiring broken periodicity. Such localized self-organization is the ILMs of discrete nonlinear systems. It is important to note that ILMs of a discrete nonlinear system are the exact eigenmodes of the nonlinear Hamiltonian describing the system. As in continuous systems, ILMs in discrete systems can also be divided in two broad categories, solitons and breathers $[7,8,9,10,11,24,26,40,44,50,54,55,60]$. In this case also the separation line is not always distinct. Consider for example the AL equation. The stationary one-soliton solutions of this equation are nothing but breathers $[1,2,3]$. ILMs are predominantly occurring nonlinear excitations in discrete nonlinear systems. To understand this, we note that stable localized modes must always be either below the band or in band gaps [22]. So, the discreteness in spatial variables can provide a favorable mechanism for the formation and the stabilization of ILMs in discrete nonlinear systems by introducing finite bandwidths and consequently accessible band edges. This in turn increases the probability that the energy of a localized self-organization in a discrete nonlinear system will lie in the band gap. Again, the band width of a perfect linear discrete system depends on the magnitude of the intersite coupling term. In a single band model, if such coupling is weak, we have a narrow band. A discrete nonlinear system with narrow bands is called anti-integrable. Such anti-integrable nonlinear systems are then expected to sustain nonlinearity-induced localized modes in the band gaps by the above argument. There is indeed a mathematical proof of this in the literature $[50,54,55]$. Of course, it is not necessary to have anti-integrable systems to have breathers. The existence of a breather solution in the N-AL equation has been shown [46]. In fact, in contrast to continuous nonlinear systems, in any general discrete nonlinear systems, particularly in nonintegrable systems, stationary breathers are predominantly occurring ILMs.

When localized states are formed below the lower edge of a band, they are unstaggered or symmetric localized states. These states are symmetric under reflection through the center, and of course low-energy localized modes of the system [13, 46]. Furthermore, these symmetric localized states can have their peak at a lattice site or in between two lattice sites. The first one is called on-site peaked unstaggered localized modes. The other one is called intersite peaked unstaggered localized modes. When localized states are formed above the upper edge of a band, they are staggered or antisymmetric localized states [13]. These states are antisymmetric under reflection and high energy excitations of a system. In case of staggered localized states, we analogously have odd-parity SieversTakeno mode (ST) as well as even-parity Page (P) mode $[58,63,65]$. It is also to be noted that staggered localized states have no analog in continuous systems [13]. Another kind of ILMs, called twisted localized modes, can be found in nonlinear lattices [17, 39]. In this category also, we can have unstaggered as well as staggered localized modes [17, 39]. When these modes are stationary modes of the system, they are called stationary localized states (SLSs). We emphasize again that SLSs of any type, if they are true eigenmodes of nonlinear systems, are also discrete breather $[24,26]$. They may be called trivial breathers.

Though it is possible to have in circumstances stationary ILMs in discrete integrable nonlinear systems, stationary ILMs are formed mostly in nonintegrable nonlinear systems. We discuss here the stationary ILMs of SLS type. We know that stationary solitons of AL equations are examples of SLSs in integrable nonlinear equations, and these are 
also breather solutions of the same equation. We should however not fail to note that though these breather solutions are band-edge states, their widths are undetermined. On the other hand, the formation of SLSs in discrete nonlinear systems depends critically on two factors, the intersite hopping term which determines the width of bands in the corresponding linear systems and the strength of the nonlinearity, which determines the energy of the self-organized localized formation. If the first term is predominant, the nonlinearity can produce at best localized modes near band edges. When localized states are formed near band edges, they are weakly localized. In other words, they have large widths and small amplitudes. Since the movement of these localized modes does not require large-scale rearrangement in the lattice, such localized modes can be made to move by applying small perturbing fields. As the movement of any unstaggered localized state will not require an inversion of orientation in any of the sites, these states can easily move compared to its staggered counterpart under small perturbation. Again, in case of unstaggered localized states, intersite peaked states will have larger widths and smaller amplitudes compared to their on-site peaked counterparts. So, intersite peaked states can be made mobile easily by a small perturbation. In the other extreme where nonlinearity is strong, strong localized modes having nonzero amplitudes only at a few sites are formed. These are of course high-energy ILMs. Odd-parity Sievers-Takeno (ST) modes and even-parity $\mathrm{P}$ modes in strongly anharmonic lattices are examples of such strongly localized modes. Since these modes are formed from the acoustic branch of anharmonic lattices, they appear above the band and hence are staggered localized states. It is further found that ST modes are unstable to an infinitesimal perturbation. However, this mode is not destroyed by the perturbation. Instead, any perturbation makes it move [39]. On the other hand, $\mathrm{P}$ mode is stable and does not move by small perturbations. The mobility difference of these modes can be understood by the PN potential. Because of the distribution of amplitudes, ST modes are formed at the maximum of the PN potential and the P modes at the bottom of this potential [16]. For the P mode to move then we need enough energy to excite this mode above the PN potential. Consequently, under a perturbation, not sufficient to take it out of the well, this mode will remain immobile. On the other hand, when ST modes are at the maximum of the PN potential, no energy is needed to take it out of the well. So, an infinitesimal perturbation can make it mobile. The mobility difference of on-site and intersite peaked unstaggered localized modes to an infinitesimal perturbation can be understood by the same argument.

With this background, I plan to study here the formation of both unstaggered and staggered stationary localized states in systems described by IN-DNLS [13]. To this end, I plan to examine the dependence of the amplitude and width of the localized modes and also the eigenfrequency of these modes on the nonintegrability parameter of the equation. The energy of the localized modes are calculated from the Hamiltonian. To the best of my knowledge, a rudimentary asymptotic analysis of this problem is done using the lattice Green function approach [13, 67]. For the detailed study, I plan to use the discrete variational approach $[5,32,48,51]$. In nonlinear dynamics, the standard variational approach has been applied to continuous nonlinear equations to study problems of nonlinear pulse propagation in optical fibers, and to soliton dynamics in massive Thirring model, to mention a few $[5,6,38,52]$. In the discrete variational approach, one directly 
proceeds to search for discrete solutions of the coupled discrete nonlinear evolution equations in a restricted subspace by imposing a suitable ansatz for the solution [5]. A procedure of averaging over the discrete dimensions leads to either a set of coupled ODDEs or a set of coupled algebraic equations or both for the solution parameters. Therefore, this approach permits one to reduce the dimension of the problem from a set of many coupled equations to generally a much smaller set of equations determined by the number of parameters in the ansatz to be determined. Clearly, this method is advantageous when the number of nonlinear equations is very large. This method has been applied to DNLS, for example, to study problems of beam steering in nonlinear waveguide arrays [5], and also to understand the formation and stability of static and dynamical solitons in onedimensional systems and Cayley trees $[32,48,51]$. We note in this context that equations like DNLS describe the evolution of canonical coordinates of the canonical phase space $[5,18]$. On the other hand, AL, N-AL, and IN-DNLS in their generic form describe the evolution of noncanonical coordinates in noncanonical phase spaces $[1,2,3,13,18,46]$. Since these equations are derivable from Hamiltonians, the geometry of the dynamics is automatically symplectic [18]. The noncanonical symplectic structure of the dynamics is manifested in the structure of the Poisson brackets $[11,12,13,46,64]$. It is, however, to be noted that there exists a global nonsingular coordinate transformation for these equations, which transforms the noncanonical coordinates to canonical coordinates [12]. Therefore, these equations can also be described by canonical coordinates with canonical Lagrangian and Poisson brackets, having canonical symplectic structure $[12,18]$. I will however proceed with the variational procedure with noncanonical coordinates. I note that in Hamiltonian dynamics, the structure of the Poisson bracket is incorporated in the Lagrangian $[12,53]$. But my analysis is done with the appropriate functional, which is also obtainable from the Lagrangian. So, the noncanonical symplectic structure of the Poisson bracket does not pose any problem of finding SLSs in IN-DNLS. The other side of this analysis is the following. It shows how the effective Lagrangian can be derived from the knowledge of the Hamiltonian and constants of motion using the analogous variational approach of finding eigenvalues in standard Sturm-Liouville problems [33]. In other words, I will also show that it is possible to set up the variational problem for the determination of eigenvalues without the prior knowledge of the Lagrangian. Finally, I note that it has been seen in continuous nonlinear equations that when the variational method is applied to analyze solitary wave dynamics, the solitary wave solutions may show instability in some range of variational parameters. On the other hand, the correct dynamics may not show at all such instability. So, the variational method can produce false instabilities $[6,51]$. This consideration also applies to discrete nonlinear evolution equations. However, I do not encounter any undesired instability in my solutions, which can be ascribed to the variational method. So, this aspect, even though important, is not dealt with here.

The organization of the paper is as follows. In Section 2 below, we present the basic equations to be studied. In Section 3, we present a set of results, coming from one particular formulation. In Section 3, we also show that our formulation gives exact stationary localized states of the AL equation. In Section 4, we present another alternative formulation of the same problem. We then present the corresponding results. Finally, we 
summarize our main results in Section 5. Besides, this paper contains three important as well as relevant appendices.

\section{Formalism}

2.1. General derivation of the nonlinear IN-DNLS equation and the variational formulation of the corresponding eigenvalue problem. We consider a dynamical system having $2 \mathrm{~N}$ generalized noncanonical coordinates, $\left\{\phi_{n}, \phi_{n}^{\star}\right\}, n=1, \ldots, N$, in a symplectic manifold [18]. Let $U$ and $V$ be any two general dynamical variables of the system. Any symplectic manifold has a natural Poisson bracket structure defined in terms of the inverse of the symplectic structure function [18]. So, we now define the following noncanonical Poisson bracket to characterize the manifold [12, 13, 64]:

$$
\{U, V\}_{\left\{\phi, \phi^{\star}\right\}}=i \sum_{n=1}^{N}\left(\frac{\partial U}{\partial \phi_{n}} \frac{\partial V}{\partial \phi_{n}^{\star}}-\frac{\partial V}{\partial \phi_{n}} \frac{\partial U}{\partial \phi_{n}^{\star}}\right)\left(1+\mu\left|\phi_{n}\right|^{2}\right) .
$$

We now consider the Hamiltonian

$$
\tilde{\mathrm{H}}=-\sum_{n}\left(\phi_{n}^{\star} \phi_{n+1}+\phi_{n+1}^{\star} \phi_{n}\right)-2 v \sum_{n}\left|\phi_{n}\right|^{2}+2 v \sum_{n} \ln \left[1+\left|\phi_{n}\right|^{2}\right]
$$

which is obtained from the original IN-DNLS Hamiltonian H through the transformations $\phi_{n} \rightarrow \sqrt{\mu} \phi_{n}, n \in Z$, and $\nu \rightarrow \nu / \mu[12,13]$. The corresponding Lagrangian $\tilde{\mathscr{L}}$ in the scaled variables $[12,53]$ is

$$
\widetilde{\mathscr{L}}=\frac{i}{2} \sum_{n}\left(\dot{\phi}_{n} \phi_{n}^{\star}-\dot{\phi_{n}^{\star}} \phi_{n}\right) \frac{\ln \left[1+\left|\phi_{n}\right|^{2}\right]}{\left|\phi_{n}\right|^{2}}-\tilde{\mathrm{H}} .
$$

The dynamical evolution of the $n$th generalized coordinate $\phi_{n}$ can then be obtained by using (2.1) and (2.2):

$$
\begin{aligned}
i \dot{\phi}_{n} & =\left(1+\left|\phi_{n}\right|^{2}\right) \frac{\partial \tilde{\mathrm{H}}}{\partial \phi_{n}^{\star}} \\
& =-\left(1+\left|\phi_{n}\right|^{2}\right)\left(\phi_{n+1}+\phi_{n-1}\right)-2 \nu\left|\phi_{n}\right|^{2} \phi_{n}
\end{aligned}
$$

for $n \in Z[12,13]$. The other set of equations is obtained by conjugation. The same equation can be obtained from the Lagrangian by using the standard Lagrangian equations of motion. We note that under the global gauge transformation $\phi_{n} \rightarrow \phi_{n} e^{i \alpha},(2.2),(2.3)$ and (2.4) remain invariant. It can also be shown from (2.4) that $\tilde{\mathcal{N}}=\sum_{n} \ln \left[1+\left|\phi_{n}\right|^{2}\right]$ is a constant of motion [13]. We now assume that $\phi_{n}=\lambda^{n} \Psi_{n} \exp (-i \omega t), n \in Z$, where $\lambda= \pm 1$. Furthermore, $\Psi_{n}, n \in Z$, are taken to be real [13]. Then from (2.4), we get

$$
(\widehat{\Omega} \hat{\Psi})_{n}=\omega \Psi_{n}+\lambda\left(1+\Psi_{n}^{2}\right)\left(\Psi_{n+1}+\Psi_{n-1}\right)+2 \nu \Psi_{n}^{3}=0
$$


This is a nonlinear eigenvalue problem and its solutions give frequencies of stationary localized states of IN-DNLS equation [13]. Introducing the above ansatz for $\phi_{n}, n \in Z$, in $\tilde{\mathcal{N}}$ and $\tilde{\mathrm{H}}$, we get

$$
\begin{aligned}
\tilde{\mathcal{N}} & =\sum_{n} \ln \left[1+\Psi_{n}^{2}\right], \\
\tilde{\mathrm{H}} & =-2 \lambda \sum_{n} \Psi_{n} \Psi_{n+1}-2 \nu \sum_{n} \Psi_{n}^{2}+2 \nu \tilde{\mathcal{N}} \\
& =\tilde{\mathrm{H}}_{0}+2 \nu \tilde{\mathcal{N}} .
\end{aligned}
$$

We define next

$$
\widetilde{\mathrm{F}}=\widetilde{\mathrm{H}}-\Lambda \widetilde{\mathcal{N}}
$$

where $\Lambda$ is the Lagrange multiplier [35]. Setting $\delta \widetilde{\mathrm{F}}=0$, we get back (2.5), when $\Lambda=\omega$. It is also important to note that the functional, $\widetilde{\mathrm{F}}$ can also be obtained from $\tilde{\mathscr{L}}$ after introducing the ansatz. In Appendix A, I plan to discuss the importance of the functional $\widetilde{\mathrm{F}}$.

2.2. Variational approach with sech ansatz. We first note that the system described by IN-DNLS equation (2.4) has lattice translational invariance. So, this system can only form ILMs, arising from the competition between the localizing nonlinearity and the dispersion from the intersite hopping [61]. As the corresponding linear system is a discrete single band system, this further enhances the propensity of formation of ILMs either below or above the band. According to the theory of localization, any self-localized state in one-dimensional systems will have exponential localization in the following sense. The amplitude $\Psi_{n}$ of the localized mode at the $n$th site will show exponential decay with $|n|$ for large values of $|n|[22,36,57]$. We should also keep in mind that a modulus function $(|\cdots|)$ cannot appear in a physical problem in its generic form. This type of functions can only be obtained in any physical problem in the asymptotic limit. Furthermore, when $\nu=0,(2.4)$ becomes the well-known AL equation $[1,2,3,13]$. The one-soliton solution of Ablowitz-Ladik (AL) equation can be either static or dynamic. For both cases, it has the sech profile, which satisfies also the other requirement for localized states in one dimension. So, we use the ansatz $\Psi_{n}=\Phi\left(1 / \cosh \beta\left(n-x_{0}\right)\right), n \in Z$. This ansatz has also been used in the previous analysis [13]. For on-site peaked and ST-like localized states, $x_{0}=0$, and for intersite peaked and P-like states, $x_{0}= \pm 1 / 2[5,58,63,65]$. We further write $\Phi^{2}=\Psi$. While $\beta^{-1}$ gives the half width of localization, $\Phi$ denotes the maximum amplitude of the states. Now, the introduction of this ansatz in the functional $\widetilde{\mathrm{F}}$ makes it an algebraic function of the parameters of the ansatz

$$
\widetilde{\mathrm{F}}\left(\Psi, \beta, \lambda, x_{0}\right)=\tilde{\mathrm{H}}\left(\Psi, \beta, \lambda, x_{0}\right)-\Lambda \tilde{\mathcal{N}}\left(\Psi, \beta, x_{0}\right)
$$

and we need to find relative extrema of $\widetilde{\mathrm{F}}$ with respect to variables $\Psi$ and $\beta$ [35]. The finding of relative extrema with respect to these two variables, $\Psi$ and $\beta$, means that $d \widetilde{\mathrm{F}}=0$ 
should imply the following equations [35]:

$$
\begin{aligned}
& \frac{\partial \tilde{\mathrm{H}}_{0}}{\partial \Psi}-\Lambda_{1} \frac{\partial \tilde{\mathcal{N}}}{\partial \Psi}=0 \\
& \frac{\partial \tilde{\mathrm{H}}_{0}}{\partial \beta}-\Lambda_{1} \frac{\partial \tilde{\mathcal{N}}}{\partial \beta}=0
\end{aligned}
$$

where $\Lambda_{1}=\Lambda-2 \nu$. For what follows, we assume that $\partial \tilde{\mathcal{N}} / \partial \Psi \neq 0$. Then from (2.10), we find that

$$
\Lambda=\omega=2 \nu+\frac{\partial \tilde{\mathrm{H}}_{0} / \partial \Psi}{\partial \tilde{\mathcal{N}} / \partial \Psi}
$$

and also

$$
f\left(\Psi, \beta, \lambda, x_{0}\right)=\left\{\tilde{\mathrm{H}}_{0}, \tilde{\mathcal{N}}\right\}_{\{\beta, \Psi\}}=0
$$

The other required equation is

$$
\tilde{\mathcal{N}}\left(\Psi, \beta, x_{0}\right)=C=\text { constant }
$$

We note that we have three unknowns, namely, $\Lambda, \Psi$, and $\beta$. But we also have three independent equations to solve these unknowns. Hence, the problem is well posed.

2.3. Calculation of $\tilde{\mathrm{H}}_{0}$ and $\tilde{\mathcal{N}}$. Introducing the expression of $\Psi_{n}, n \in Z$, in $\tilde{\mathrm{H}}_{0}$ and $\tilde{\mathcal{N}}$ we get

$$
\tilde{\mathrm{H}}_{0}\left(\Psi, \beta, \lambda, x_{0}\right)=-2 \lambda \Psi S_{1}\left(\beta, x_{0}\right)-2 \nu \Psi S_{2}\left(\beta, x_{0}\right),
$$

where

$$
\begin{aligned}
& S_{1}\left(\beta, x_{0}\right)=\sum_{n=-\infty}^{\infty} \frac{1}{\cosh \beta\left(n-x_{0}\right) \cosh \beta\left(n+1-x_{0}\right)}, \\
& S_{2}\left(\beta, x_{0}\right)=\sum_{n=-\infty}^{\infty} \frac{1}{\cosh ^{2} \beta\left(n-x_{0}\right)}, \\
& \tilde{\mathcal{N}}\left(\Psi, \beta, x_{0}\right)=\sum_{n=-\infty}^{\infty} Y_{n}\left(\Psi, \beta, x_{0}\right),
\end{aligned}
$$

where

$$
Y_{n}\left(\Psi, \beta, x_{0}\right)=\ln \left[1+\frac{\Psi}{\cosh ^{2} \beta\left(n-x_{0}\right)}\right]
$$


604 A discrete variational approach for investigation in IN-DNLS

To evaluate $S_{1}\left(\beta, x_{0}\right), S_{2}\left(\beta, x_{0}\right)$, and $\tilde{\mathcal{N}}\left(\Psi, \beta, x_{0}\right)$, we make use of the famous Poisson sum formula $[5,45,46,70,72]$ which reads

$$
\sum_{n=-\infty}^{\infty} f(n \beta)=\frac{1}{\beta} \int_{-\infty}^{\infty} d y\left[1+2 \sum_{s=1}^{\infty} \cos \left(\frac{2 \pi s y}{\beta}\right)\right] f(y) .
$$

This application yields

$$
\begin{gathered}
S_{1}\left(\beta, x_{0}\right)=\frac{2}{\sinh \beta}, \\
S_{2}\left(\beta, x_{0}\right)=\frac{2}{\beta}+\frac{4}{\beta} \sum_{s=1}^{\infty} \Gamma_{s}\left(\beta, x_{0}\right), \\
\Gamma_{s}\left(\beta, x_{0}\right)=\cos 2 \pi s x_{0} \frac{\pi^{2} s / \beta}{\sinh \left(\pi^{2} s / \beta\right)} ; \\
\sqrt{\Psi(1+\Psi)} \frac{\partial \tilde{\mathcal{N}}}{\partial \Psi}=\frac{2}{\beta} \operatorname{arcsinh} \sqrt{\Psi}+\frac{2 \pi}{\beta} \sum_{s=1}^{\infty} T_{s}(\Psi, \beta) \cos 2 \pi s x_{0},
\end{gathered}
$$

where

$$
T_{s}(\Psi, \beta)=\frac{\sin [(2 \pi s / \beta) \operatorname{arcsinh} \sqrt{\Psi}]}{\sinh \left(\pi^{2} s / \beta\right)} .
$$

We now define the function, $f_{1}\left(\beta, \nu, \lambda, x_{0}\right)$

$$
f_{1}\left(\beta, \nu, \lambda, x_{0}\right)=\frac{\sinh \beta}{1+\lambda \nu(\sinh \beta / \beta) S_{3}\left(\beta, \nu, \lambda, x_{0}\right)},
$$

where

$$
S_{3}\left(\beta, \nu, \lambda, x_{0}\right)=1+2 \sum_{s=1}^{\infty} \cos 2 \pi s x_{0} \frac{\pi^{2} s / \beta}{\sinh \left(\pi^{2} s / \beta\right)} .
$$

Now, with this definition, we have

$$
\begin{gathered}
\tilde{\mathrm{H}}_{0}=-4 \lambda \frac{\Psi}{f_{1}\left(\beta, \nu, \lambda, x_{0}\right)}, \\
\frac{\partial \tilde{\mathrm{H}}_{0}}{\partial \Psi}=-\frac{4 \lambda}{f_{1}\left(\beta, \nu, \lambda, x_{0}\right)}, \\
\frac{\partial \tilde{\mathrm{H}}_{0}}{\partial \beta}=-4 \lambda \Psi \frac{\partial\left(1 / f_{1}\left(\beta, \nu, \lambda, x_{0}\right)\right)}{\partial \beta} .
\end{gathered}
$$


Again from (2.20), we have

$$
\tilde{\mathcal{N}}\left(\Psi, \beta, x_{0}\right)=\frac{2}{\beta}(\operatorname{arcsinh} \sqrt{\Psi})^{2}+4 \sum_{s=1}^{\infty} \cos 2 \pi s x_{0} \frac{\sin ^{2}((\pi s / \beta) \operatorname{arcsinh} \sqrt{\Psi})}{s \sinh \left(\pi^{2} s / \beta\right)},
$$

and from (2.27) we in turn get

$$
\begin{aligned}
\frac{\partial \tilde{\mathcal{N}}}{\partial \beta}= & -\frac{2}{\beta^{2}}(\operatorname{arcsinh} \sqrt{\Psi})^{2}-\frac{4 \pi \operatorname{arcsinh} \sqrt{\Psi}}{\beta^{2}} \sum_{s=1}^{\infty} \cos 2 \pi s x_{0} \frac{\sin ((2 \pi s / \beta) \operatorname{arcsinh} \sqrt{\Psi)}}{\sinh \left(\pi^{2} s / \beta\right)} \\
& +\frac{4 \pi^{2}}{\beta^{2}} \sum_{s=1}^{\infty} \cos 2 \pi s x_{0} \frac{\sin ^{2}((\pi s / \beta) \operatorname{arcsinh} \sqrt{\Psi})}{\sinh \left(\pi^{2} s / \beta\right)} \operatorname{coth} \frac{\pi^{2} s}{\beta} .
\end{aligned}
$$

The calculation of (2.27) is given in Appendix B.

In our variational formulation, in principle $x_{0}$ is another parameter to be determined from the extrema of the functional $\widetilde{\mathrm{F}}(2.8)$. Now, the extremization of $\widetilde{\mathrm{F}}$ with $x_{0}$ inclusive will yield, along with (2.10), the following equation:

$$
\frac{\partial \tilde{\mathrm{H}}_{0}}{\partial x_{0}}-\Lambda_{1} \frac{\partial \tilde{\mathcal{N}}}{\partial x_{0}}=0
$$

But, from (2.22), (2.24), and (2.27), it can be easily proved that as $0 \leq\left|x_{0}\right|<1, x_{0}=$ $0, \pm 1 / 2$.

\section{The variational formulation with $\tilde{\mathcal{N}}$ constant : results and discussion}

3.1. Ablowitz-Ladik limit. In the Ablowitz-Ladik limit, $v=0$. To probe this limit, we evaluate relevant functions and their derivatives along the curve $\Psi=\sinh ^{2} \beta$. Along this curve, from (2.20), (2.27), and (2.28) we have

$$
\begin{gathered}
\tilde{\mathcal{N}}\left(\Psi, \beta, x_{0}\right)=2 \beta, \\
\frac{\partial \tilde{\mathcal{N}}}{\partial \beta}=-2, \\
\frac{\partial \tilde{\mathcal{N}}}{\partial \Psi}=\frac{2}{\sinh \beta \cosh \beta} .
\end{gathered}
$$

Since $v=0$ in this case, we also have from (2.22)-(2.26)

$$
\begin{aligned}
& \frac{\partial \tilde{\mathrm{H}}_{0}}{\partial \Psi}=-\frac{4 \lambda}{\sinh \beta}, \\
& \frac{\partial \tilde{\mathrm{H}}_{0}}{\partial \beta}=4 \lambda \cosh \beta
\end{aligned}
$$


We find from (3.2)-(3.5) that $f\left(\Psi, \beta, \lambda, x_{0}\right)=\left\{\tilde{\mathrm{H}}_{0}, \tilde{\mathcal{N}}_{\{\beta, \Psi\}}=0\right.$. Furthermore, from (2.11), (3.3), and (3.4) we get $\omega=-2 \lambda \cosh \beta$. The energy $\widetilde{\mathrm{E}}=\tilde{\mathrm{H}}=-4 \lambda \sinh \beta$. Due to positive semidefiniteness of $\widetilde{\mathcal{N}}$, we get from (3.1) that $\beta$ should also be positive semidefinite. This is consistent with the one-soliton solution of Ablowitz-Ladik equation.

We now consider the case when $v \neq 0$. For convenience, we define

$$
g\left(\beta, x_{0}\right)=\frac{1}{\beta}\left[1+2 \sum_{s=1}^{\infty} \cos 2 \pi s x_{0} \frac{\pi^{2} s / \beta}{\sinh \left(\pi^{2} s / \beta\right)}\right] .
$$

Along the line $\Psi=\sinh ^{2} \beta$, we find that

$$
f\left(\Psi, \beta, \lambda, x_{0}\right)=\left\{\tilde{\mathrm{H}}_{0}, \tilde{\mathcal{N}}\right\}_{\{\beta, \Psi\}}=-8 v g\left(\beta, x_{0}\right) \tanh \beta \frac{d \ln A_{0}\left(\beta, x_{0}\right)}{d \beta},
$$

where $A_{0}\left(\beta, x_{0}\right)=\sinh \beta g\left(\beta, x_{0}\right)$. When $\beta \rightarrow 0, \tanh \beta\left(d \ln A_{0}\left(\beta, x_{0}\right) / d \beta\right) \rightarrow \beta^{2} / 3$ and consequently $f\left(\Psi, \beta, \lambda, x_{0}\right) \sim-(8 \nu / 3) \beta^{2}$, provided $\nu$ is finite. So, when $\left(\nu \beta^{2}\right) \sim o(1), \Psi=$ $\sinh ^{2} \beta$ is an asymptotic solution of a localized state with a large width and a small amplitude. Eigenvalue $\omega$ and energy $\widetilde{\mathrm{E}}=\tilde{\mathrm{H}}$ of these localized states are

$$
\begin{aligned}
\omega & =2 \nu-2\left(\lambda+\nu A_{0}\right) \cosh \beta \\
& \sim-2 \lambda-\left(\lambda+\frac{4 \nu}{3}\right) \beta^{2}, \\
\widetilde{\mathrm{E}} & =-4 \lambda \beta-\frac{2}{3} \beta^{3}-4 \nu \beta\left[\frac{A_{0} \sinh \beta}{\beta}-1\right] \\
& \sim-4 \lambda \beta-\frac{2}{3}(\lambda+2 \nu) \beta^{3} .
\end{aligned}
$$

So, according to this asymptotic analysis, when $v \neq 0$, the nonintegrability parameter $v$ and the width parameter $\beta$ of the SLS are not independent of each other.

3.2. Stationary localized states from IN-DNLS. We now consider various mathematical aspects of the formation of stationary localized states in IN-DNLS. We consider first (2.13) along with (2.27). We restrict ourselves to $\beta \geq 0$, which is necessary to keep $\Psi$ positive semidefinite. Furthermore, in the following analysis, we assume that $\beta \leq 1$. In this situation, we can ignore infinite sums in (2.22) and in (2.27). Due to this approximation, (2.27) yields $\Psi=\sinh ^{2} \alpha \sqrt{\beta}$ where $\alpha$ is a constant, as required by (2.13). Since the right-hand side of (2.13) is taken to be a number constant $C=2.0 \alpha^{2}$, we have $d \tilde{\mathcal{N}} / d \beta=0$ irrespective of the value of $\beta$. This in turn gives

$$
\frac{d \Psi}{d \beta}=-\frac{\partial \tilde{\mathcal{N}} / \partial \beta}{\partial \tilde{\mathcal{N}} / \partial \Psi}
$$

Now introducing (3.9) in (2.12), we get $d \tilde{\mathrm{H}}_{0} / d \beta=0$. In other words, permissible values of $\beta$ are determined from the extrema of $\tilde{\mathrm{H}}_{0}$ as a function of $\beta$. From the functional 


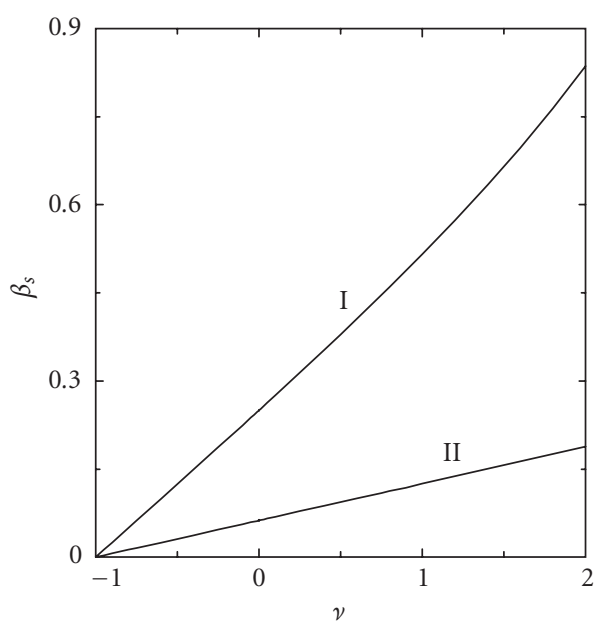

Figure 3.1. The variation of the smaller $\operatorname{root} \beta_{s}$ of (3.10) as a function of the nonintegrability parameter $\nu$. Since $\lambda=1$, these states are unstaggered stationary localized states. Curve I: $\alpha=0.5$ and curve II: $\alpha=0.25$.

dependence of $\tilde{\mathrm{H}}_{0}$ and $\Psi$ on $\beta$, we ultimately get

$$
\begin{gathered}
g_{1}(\alpha, \beta)=\frac{\beta}{\sinh \beta} \cosh \beta \frac{\tanh \alpha \sqrt{\beta}}{\alpha \sqrt{\beta}}-1, \\
g_{2}(\alpha, \beta)=1-\frac{\tanh \alpha \sqrt{\beta}}{\alpha \sqrt{\beta}}, \\
\nu \lambda=\frac{\beta}{\sinh \beta} \frac{g_{1}(\alpha, \beta)}{g_{2}(\alpha, \beta)} .
\end{gathered}
$$

We note that for a given value of the parameter $\alpha, \beta$ is determined by the nonintegrability parameter, $\nu$. Furthermore, (3.10) yields two positive values of $\beta$ as roots, under two conditions, namely, $\nu \lambda \geq 0$ and $|\nu|<\left|\nu_{\text {critical }}\right|$. The behavior of the smaller root $\left(\beta_{s}\right)$ as a function of $\nu$ for $\lambda=1$ and $\alpha=0.5$ and 0.25 are shown in Figure 3.1. In Figure 3.2, we present the variation of $\beta_{s}$ as a function of the parameter $\alpha$ for various values of $\nu \geq 0$. It should be noted from these figures that $\beta_{s} \leq 1$ for these values of $\alpha$ and the chosen interval of $\nu$. So, the neglect of infinite sums in (2.22) and (2.27) is justified. It is a simple exercise to see from (3.10) that when $|\nu| \rightarrow 0, \beta_{s} \rightarrow \alpha^{2}$. Then, for small values of $\nu$ the asymptotic solution is the AL stationary localized state solution. This is a very important result. This asymptotic analysis reveals that this stationary localized state solution of IN-DNLS continuously moves to the AL stationary localized state solution when $\nu \rightarrow 0$ from either side. It is further important to note from Figure 3.2 that for $\alpha \ll 1$, we have $\alpha \approx \sqrt{\beta_{s} /(1.0+\nu \lambda)}, \nu \lambda \geq 0$. Consequently, $\sqrt{\Psi} \approx \sinh \left(\beta_{s} / \sqrt{(1.0+\nu \lambda)}\right)$. But, as for this range of argument, $\sinh x \approx x$, we have $\sqrt{\Psi} \sim \beta_{s} / \sqrt{1.0+v \lambda}$. This agrees with the existing asymptotic analysis [13]. 


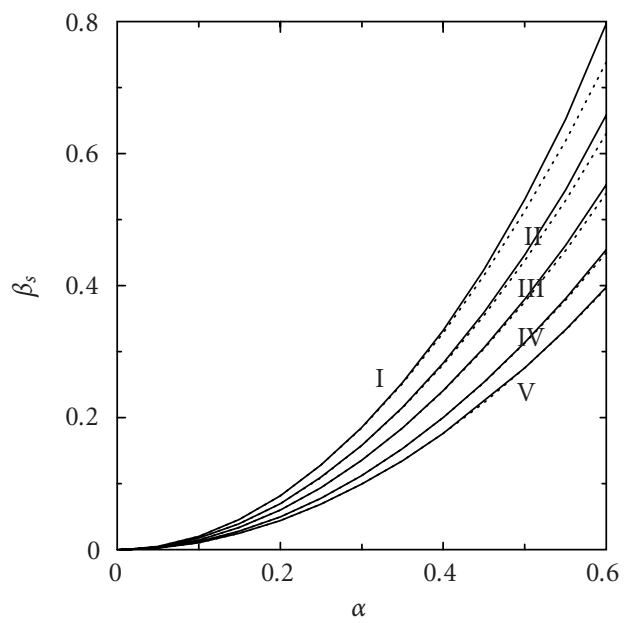

Figure 3.2. The variation of the smaller root $\beta_{s}$ of (3.10) as a function of the parameter $\alpha$ for various values of the nonintegrability parameter $\nu$. Since $\lambda=1$, these states are unstaggered stationary localized states. Curve I: $v=1.05$, curve II: $\nu=0.75$, curve III: $v=0.5$, curve IV: $v=0.25$, and curve $\mathrm{V}: \nu=0.10$. Each curve is associated with a dotted curve which shows the variation of $\alpha^{2}(1+\nu \lambda)$ as a function of $\alpha$ for the corresponding value of $\nu$.

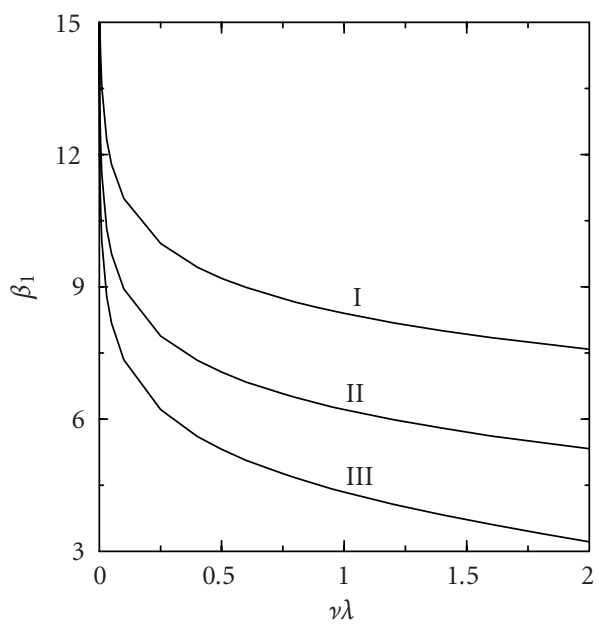

Figure 3.3. The variation of the larger $\operatorname{root} \beta_{l}$ of (3.10) as a function of $\nu \lambda$. If $\lambda=1$, these states are then unstaggered stationary localized states. Curve I : $\alpha=0.1$, curve II: $\alpha=0.25$, and curve III: $\alpha=0.50$.

The variation of the large root $\beta_{l}$ as a function of $\nu \lambda$ for various values of $\alpha$ is shown in Figure 3.3. Again, by comparing Figures 3.1 and 3.3, we see that as $v$ increases, the large root $\beta_{l}$ of (3.10) decreases from $\infty$, while the other root $\beta_{s}$ increases from zero. So, for a given $\alpha$, the value of $\nu_{\text {critical }}$ is determined by the inflection point of $\tilde{\mathrm{H}}_{0}$. This then implies that the equations used to solve $\beta_{\text {critical }}$ and $\nu_{\text {critical }}$ are obtained by setting 


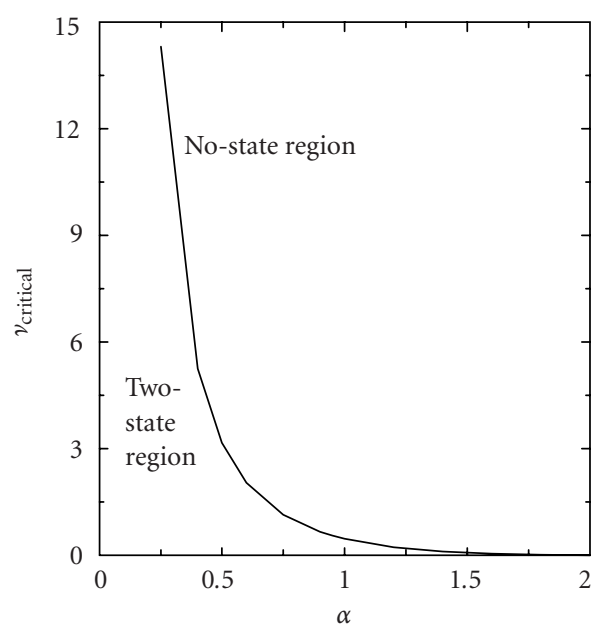

Figure 3.4. The variation of $v_{\text {critical }}$ as a function of the parameter $\alpha$ for unstaggered stationary localized states $\lambda=1 . v_{\text {critical }}$ is obtained from (3.10) and (3.11) in the text. Note that the curve separates the two-state region from the no-state region.

both $d \tilde{\mathrm{H}}_{0} / d \beta=0$ and $d^{2} \tilde{\mathrm{H}}_{0} / d \beta^{2}=0$. While the first condition gives (3.10), the second condition yields (3.11) as shown below:

$$
\begin{gathered}
g_{3}(\beta)=\frac{\tanh \sqrt{\beta}}{\sqrt{\beta}}, \quad g_{4}(\beta)=\frac{\beta}{\sinh \beta}, \\
g_{5}(\alpha, \beta)=1-g_{3}\left(4 \alpha^{2} \beta\right)-4 g_{3}\left(4 \alpha^{2} \beta\right)\left(1-g_{3}\left(\alpha^{2} \beta\right)\right), \\
g_{6}(\alpha, \beta)=1-g_{3}\left(4 \alpha^{2} \beta\right)\left(1+4 \cosh \beta g_{4}(\beta)\right), \\
g_{7}(\alpha, \beta)=g_{4}(\beta) g_{3}^{2}\left(\alpha^{2} \beta\right)\left(\beta^{2}+2 g_{4}^{2}(\beta)\right), \\
g_{8}(\alpha, \beta)=-2 \nu \frac{\alpha^{2}}{\beta^{2}} \cosh 2 \alpha \sqrt{\beta} g_{5}(\alpha, \beta), \\
g_{9}(\alpha, \beta)=-2 \lambda \frac{\alpha^{2}}{\beta^{2}} \cosh 2 \alpha \sqrt{\beta} g_{4}(\beta) g_{6}(\alpha, \beta), \\
g_{10}(\alpha, \beta)=-4 \lambda \frac{\alpha^{2}}{\beta^{2}} \cosh ^{2} \alpha \sqrt{\beta} g_{7}(\alpha, \beta), \\
g_{8}(\alpha, \beta)+g_{9}(\alpha, \beta)+g_{10}(\alpha, \beta)=0 .
\end{gathered}
$$

We again note that $\lambda= \pm 1$ and $\nu$ in (3.11) is given by (3.10). Of course, $\nu \lambda$ is positive. We find from (3.11) that when $\alpha \rightarrow 0, v_{\text {critical }} \rightarrow \infty$. Again, when $\alpha \gg 1, v_{\text {critical }} \sim 0$. The functional dependence of $v_{\text {critical }}$ on $\alpha$ is shown in Figure 3.4.

The other important case is when $\nu \lambda<0$. This means that we have either an unstaggered state with $-\nu$ or a staggered state with $+\nu$. In this case if $|\nu \lambda|>1$, both roots of $(3.10)$ are negative. Inasmuch as $\widetilde{\mathcal{N}}\left(\psi, \beta, x_{0}\right)$ is positive semi-definite, it is easy to see from $(2.27)$ that this is not permissible. For this case, from (3.10) expectedly we obtain that when 


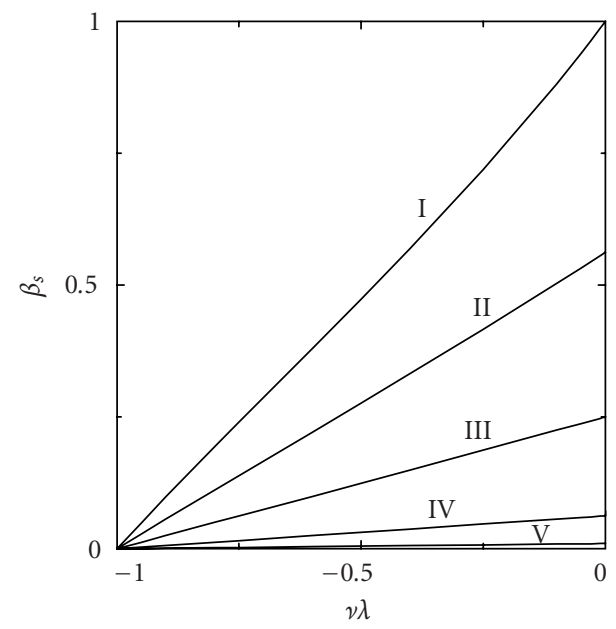

Figure 3.5. The variation of the smaller root $\beta_{s}$ of (3.10) as a function of $\nu \lambda$ for $\nu \lambda<0$ for various values of the nonintegrability parameter $\alpha$. For $\lambda=-1$, these states are staggered stationary localized states. Curve I: $\alpha=1.0$, curve II: $\alpha=0.75$, curve III: $\alpha=0.5$, curve IV: $\alpha=0.25$, and curve V: $\alpha=0.10$.

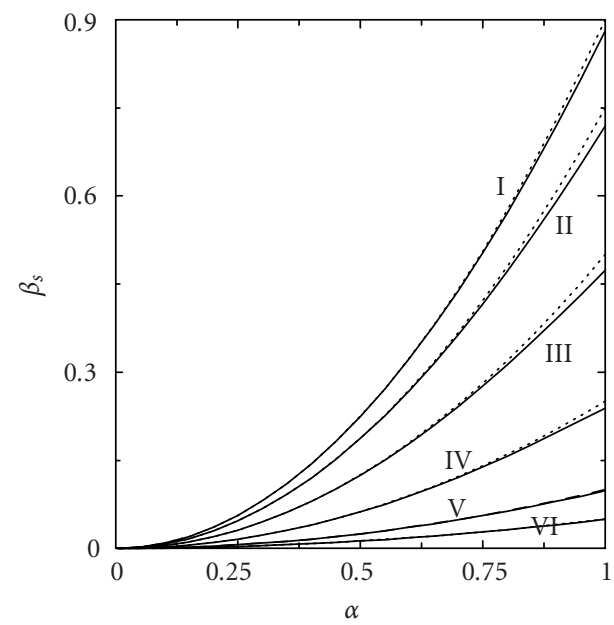

Figure 3.6. The variation of the smaller $\operatorname{root} \beta_{s}$ of (3.10) as a function of the parameter $\alpha$ for various values of the nonintegrability parameter $\nu$. Since $\lambda=-1$, these states are staggered stationary localized states. Curve I: $v=0.1$, curve II: $v=0.25$, curve III: $v=0.5$, curve IV: $v=0.75$, curve V: $v=0.90$, and curve VI: $\nu=0.95$. Each curve is also associated as in Figure 3.2 with a dotted curve which shows the variation of $\alpha^{2}(1+\nu \lambda)$ as a function of $\alpha$ for the corresponding value of $\nu$.

$\nu \lambda \rightarrow-1+, \beta \rightarrow 0$, and when $\nu \lambda \rightarrow 0-, \beta \rightarrow \alpha^{2}$. See both Figures 3.1 and 3.5. Inasmuch as for $\alpha \leq 1$, permissible values of $\beta_{s} \leq 1$, the neglect of infinite sums in (2.22) and (2.27) is again well justified. The variation of $\beta_{s}$ as a function of $\nu$ for $\alpha=1.0,0.75,0.5,0.25$, and 0.10 is shown in Figure 3.5. It is seen from Figure 3.6 that when $\nu \lambda<0, \alpha=\sqrt{\beta_{s} /(1.0+\nu \lambda)}$ 


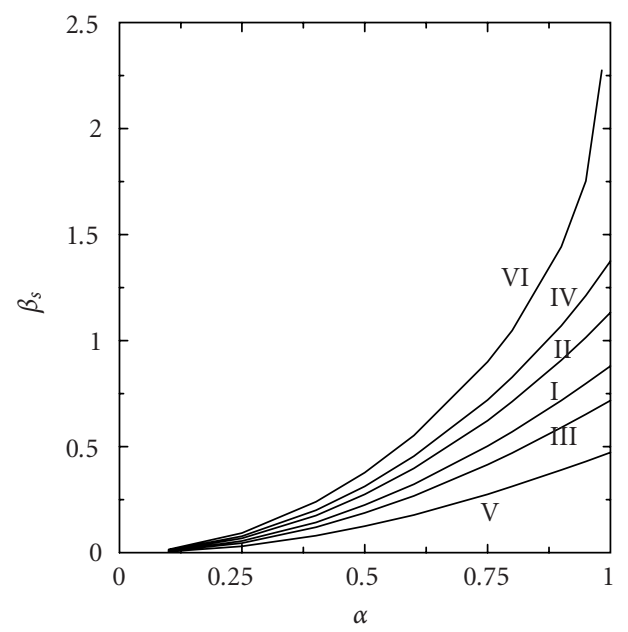

Figure 3.7. Comparison of the variation of the smaller root $\beta_{s}$ of (3.10) for both unstaggered $(\lambda=1)$ and staggered $(\lambda=-1)$ stationary localized states as a function of the parameter $\alpha$ for various values of the nonintegrability parameter $\nu$. Curve I: $\nu \lambda=-0.1$, curve II: $\nu \lambda=0.1$, curve III: $\nu \lambda=-0.25$, curve IV: $\nu \lambda=0.25$, curve V: $\nu \lambda=-0.5$, and curve VI: $\nu \lambda=0.5$.

is a very good approximation [13]. Another important aspect is in Figure 3.7, which shows that for a given $\nu>0$, the staggered SLS $(\lambda=-1)$ has larger width than the corresponding unstaggered SLS $(\lambda=1)$. So, the SLSs for $\nu \lambda<0$ are basically localized states with large widths and small amplitudes. As for eigenvalues of these stationary localized states, introducing (2.20) and (2.25) into (2.11) and using the same approximation as used for finding the roots of (3.10), we obtain that

$$
\omega=-2 \nu\left[\frac{\sinh 2 \alpha \sqrt{\beta}}{2 \alpha \sqrt{\beta}}-1\right]-2 \lambda \frac{\beta}{\sinh \beta} \frac{\sinh 2 \alpha \sqrt{\beta}}{2 \alpha \sqrt{\beta}} .
$$

The energy of these stationary states is given by

$$
\widetilde{\mathrm{E}}=\tilde{\mathrm{H}}=\widetilde{\mathrm{H}}_{0}+2 \nu \tilde{\mathcal{N}}=-4 \lambda \frac{\sinh ^{2} \alpha \sqrt{\beta}}{\sinh \beta}-4 \nu \alpha^{2}\left[\frac{\sinh ^{2} \alpha \sqrt{\beta}}{\alpha^{2} \beta}-1\right] .
$$

$\beta$ in (3.12) and (3.13) is the root of (3.10). For $\beta=\beta_{s}$ and $\alpha$ not too large, these equations are well justified. We already noted that when $\nu=0, \beta_{s}=\alpha^{2}$. Furthermore, $|\nu \lambda| \ll 1$ and also $\alpha \ll 1, \beta_{s} \rightarrow \alpha^{2}$. We obtain the respective limiting results for these cases from (3.12) and (3.13).

3.3. Stability and position of stationary localized states of IN-DNLS. We now discuss the issue of stability of these stationary localized states. We note first that when $v=0$, the resulting nonlinear equation is the AL equation, which has both unstaggered and staggered stationary localized states. These are basically band-edge states. Our variational calculation correctly produces these states of the AL equation, by letting $\beta_{s} \rightarrow \alpha^{2}$ as $|\nu| \rightarrow 0$. 
See Figures 3.1 and 3.5. Over and above it suggests another state for which $\beta_{l}=\infty$. This can be easily seen in (3.10). It is again seen from Figures 3.1 and 3.2 that for any $\alpha$, the introduction of any $\nu$, however small, with $\nu \lambda>0$, makes $\beta_{s}>\alpha^{2}$. We observe that $\beta_{s}^{-1}$ gives the half-width of the localized state. So, for these localized states, the half-width reduces with increasing $\nu$. Since $\lambda=1$ and $\nu>0(\nu \lambda>0)$ imply that the on-site nonlinear trapping potential is attractive, any positive enhancement of $\nu$ should reduce the half-width of the SLS by effectively reducing the intersite hopping. Whether a given $\nu$ defines an attractive or a repulsive potential also depends upon the value of $\lambda$. So, the above argument will hold good whenever $\nu \lambda>0$. When $\nu \lambda$ is positive, unstaggered stationary localized states characterized by $\beta_{s}$ are stable. On the other hand, for $\nu=0$, if there is any stationary localized state corresponding to $\beta_{l}=\infty$, it is a state with a peak of infinite height at a given site with a half-width of a few sites. Again, we see from Figure 3.3 that for any $\alpha$, when $\nu \lambda$ increases, $\beta_{l}$ decreases. This means that the half-width increases. On the other hand, the introduction of $\nu$ with $\nu \lambda>0$ should reduce the half-width as our argument suggests. Hence, stationary localized states corresponding to $\beta_{l}$ are unstable. These states, if existing in this system, will be unstable towards perturbation.

Consider next the case where $\nu \lambda<0$. In this case, we have either staggered localized states for positive $\nu$ or unstaggered localized states for negative $\nu$. First of all, there is only one set of stationary localized states. Furthermore, $0 \leq \beta_{s} \leq \alpha^{2}$ for $-1 \leq \nu \lambda \leq 0$. See Figure 3.5. Since, for $\nu>0$, staggered localized states are stabilized by increasing the halfwidth (see Figure 3.7), states characterized by $\beta_{s}$ are stable. For $\nu$ negative and $\lambda=1$, or $\nu>0$ and $\lambda=-1$, the on-site nonlinear potential is repulsive. So, the expansion of the half-width with decreasing $v$ is energetically favorable (see Figure 3.7).

So far our analysis did not include the effect of $x_{0}$, the position of the peak on the formation of stationary localized states and their stability. But this is also an important part of the problem. However, even a semi-rigorous investigation of this problem in this formulation requires the analytical solution of $\Psi$ as exactly as possible from (2.27). As there is no simple analytical way of solving (2.27) for $\Psi$, one can take recourse to approximation methods like the method of successive substitutions [34]. I will describe in Appendix $\mathrm{C}$ how this method can be used to get approximate dependence on $x_{0}$, of $\beta, \omega$, and $E$ of the SLS. Of course, the other possibility is to find real positive roots of $(2.27)$ graphically. Inasmuch as the exact analytical solution of $\Psi$ as a function of $\beta$ is difficult in this approach, we will not follow the present line of investigation further. On the contrary, we will show next how the exact dependence of the parameter $\beta$, the frequency $\omega$, and the energy $E$ of the SLS on $x_{0}$ can be obtained by a rational alternation in the variational procedure.

\section{The variational formulation with $\tilde{\mathrm{H}}_{0}$ constant: results and discussion}

Since $\tilde{H}$ and $\tilde{\mathcal{N}}((2.2)$ and (2.6), resp.) are two constants of motion, from the expression of $\tilde{\mathrm{H}}$, we see that $\tilde{\mathrm{H}}_{0}(2.2)$ is also a constant of motion. So, we reformulate in this section our variational problem in which $\tilde{\mathrm{H}}_{0}$, in lieu of $\tilde{\mathcal{N}}$, is taken to be the number constant. In this modified variational approach, we take $\widetilde{\mathrm{F}}=\Lambda_{2} \tilde{\mathrm{H}}_{0}+2 \nu \tilde{\mathcal{N}}$, where $\left(\Lambda_{2}-1\right)$ is the Lagrange multiplier. This modified approach also yields (2.11) for the eigenvalue, $\omega$ in (2.5). One other equation required to solve one of the two unknowns, namely, $\beta$ and $\Psi$, is given by 
(2.12). These results are also derived in Appendix A. From (2.24) we find that

$$
\Psi\left(\beta, \nu, \lambda, x_{0}\right)=a f_{1}\left(\beta, \nu, \lambda, x_{0}\right)
$$

where $a$ is a number constant, yields $\tilde{\mathrm{H}}_{0}=-4 \lambda a$, which is again a number constant. Most importantly, in this formulation $\Psi$ is determined explicitly in terms of $\beta$ within a multiplicative number constant $a$. Inasmuch as $\Psi$ is positive semi-definite by definition, the sign of this constant should be such that $a f_{1}$ is positive semi-definite. Furthermore, in this approach (2.24) yields $d \tilde{\mathrm{H}}_{0} / d \beta=0$, irrespective of $\beta$. This in turn gives

$$
\frac{d \Psi}{d \beta}=-\frac{\partial \tilde{\mathrm{H}}_{0} / \partial \beta}{\partial \tilde{\mathrm{H}}_{0} / \partial \Psi}=\Psi \frac{\partial \ln f_{1}\left(\beta, \nu, \lambda, x_{0}\right)}{\partial \beta},
$$

where we have used (2.25) and (2.26). Equation (4.2) can also be obtained from (4.1). Now introducing (4.2) in (2.12), we get $d \tilde{\mathcal{N}} / d \beta=0$. In other words, permissible values of $\beta$ are determined from the extrema of $\tilde{\mathcal{N}}$, as a function of $\beta$. The determination of extrema in turn needs (4.2), (2.20), and (2.28).

Before we proceed further, we observe the following. Here, we have a variation problem involving two variables, $\Psi$ and $\beta$. When $\Psi$ is expressed as a function of $\beta$, we obtain the Hamiltonian $\tilde{\mathrm{H}}=\tilde{\mathrm{H}}(\beta)$, and SLSs are determined from its extrema, which are obtained by setting $d \tilde{\mathrm{H}} / d \beta=0$. Of course, instead of $\beta$, we could have used $\Psi$ as the fundamental variable. Now, when $\tilde{\mathcal{N}}$ is constant, the structure of $\tilde{\mathrm{H}}(2.7)$ is such that its extrema are determined from the extrema of $\tilde{\mathrm{H}}_{0}$. We have already investigated here this part. On the other hand, we also have the option to take $\tilde{\mathrm{H}}_{0}$ to be constant, as it is done in this section and in sections to follow. In this limit the extrema of $\tilde{\mathrm{H}}(2.7)$ are determined from the extrema of $\tilde{\mathcal{N}}$, provided $\tilde{\mathcal{N}}(\beta)$ has extrema. Another equivalent way of envisioning the problem comes from (2.12), which is of course the direct consequence of the structure of the Hamiltonian $\tilde{\mathrm{H}}$ (2.7). We can think of an effective dynamical system having two conjugate dynamical variables, $\beta$ and $\Psi$. Then, the Poisson bracket formula (2.12) suggests that the effective or the reduced dynamical system can be described by the Hamiltonian $\tilde{\mathrm{H}}_{0}(\Psi, \beta)$ having a constant of motion $\tilde{\mathcal{N}}(\Psi, \beta)$, or vice versa. Stationary localized states in this dynamical system picture are determined by fixed points (FPs) of the effective or the reduced dynamical system. The two sets of extrema obtained from two procedures or two pictures may not be identical. So, in the following section, I investigate this aspect of the problem.

4.1. Equation for the fixed points of the reduced dynamical system and results. Now, if we altogether ignore the infinite sum in (2.27) which defines $\widetilde{\mathcal{N}}\left(\Psi, \beta, x_{0}\right)$, we obtain

$$
\sqrt{\Psi}=\sinh \left[\frac{\beta}{\sqrt{\Psi(1+\Psi)}} \frac{d \Psi}{d \beta}\right] .
$$

Introducing (4.1) and (4.2) in (4.3), we get the equation which determines $\beta$. If we are mostly interested in the roots of (4.3), having magnitude less than unit magnitude, we 


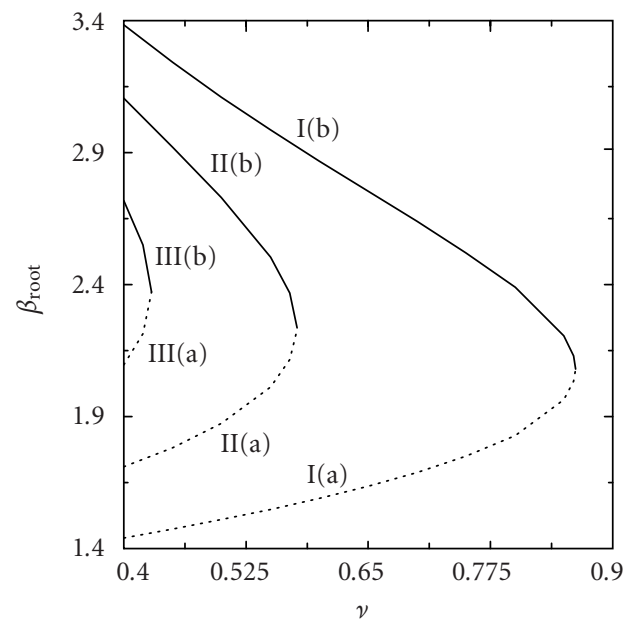

Figure 4.1. Two real roots of (4.3) in the text as a function of the nonintegrability parameter $v$ for three values of the parameter $a$. Since $\lambda=1$, these states are unstaggered stationary localized states. Curves I(a) and I(b): $a=1.5$, curves II(a) and II(b): $a=1.75$, and curves III(a) and III(b) $a=2.0$. While (a) or the lower part of all curves is for the smaller root $\beta_{s}$, (b) or the upper part of these curves show the variation of the larger root $\beta_{l}$. Note that this figure also shows the variation of $\nu_{\text {critical }}$ as a function of the parameter $a$.

can as before ignore altogether the infinite sum in (2.22), which defines $f_{1}\left(\beta, \nu, \lambda, x_{0}\right)$. This further simplifies the equation, which determines $\beta$.

When $v=0$, using (2.22) and (4.1) we find that $\beta_{s}=\operatorname{arcsinh} a$ makes the (4.3) an identity. We will later show that even with the full expression of $d \tilde{\mathcal{N}} / d \beta$, derivable from (2.27), the above choice of $\beta_{s}$ makes $d \tilde{\mathcal{N}} / d \beta$ identically zero. In other words, irrespective of $\widetilde{\mathcal{N}}$ or $\tilde{\mathrm{H}}_{0}$ taken to be a constant in this constrained variational approach, we get the same stationary AL solitons with $\omega=-2 \lambda \cosh \beta$ in both cases. Furthermore, when $\nu \sim o(1)$, we expect from this result that $\beta_{s} \sim \operatorname{arcsinh} a$. This is also borne out in our numerical calculation, albeit not shown here. We will show it in the exact calculation.

Considering (4.3), we consider first the unstaggered localized states with $v>0$. First of all, for every value of $a>0$, we find a $v_{\text {critical }}$, such that for $v>v_{\text {critical }},(4.3)$ has no real root. On the other hand, $v<v_{\text {critical }}(a)$, we find two roots of (4.3) for a given value of $a>0$. It is also found that $\beta_{s}$ is a monotonically increasing function of $\nu$ while $\beta_{l}$ is a monotonically decreasing function of $\nu$. These features of the solutions are shown in Figure 4.1. Then, according to our previous discussion, stationary localized states characterized by $\beta_{s}$ are stable, while the states characterized by $\beta_{l}$ are unstable. In case of staggered localized states having $\nu>0$, we find that for $|\nu \lambda|>1$, (4.3) has no root. Furthermore, $0<|\nu \lambda|<1,(4.3)$ has only one root, $\beta_{s}$. We find for $\lambda=-1$ that $\beta_{s}$ decreases with increasing $\nu$. This is shown in Figure 4.2. Since $\beta_{s}^{-1}$ gives a measure of the width of the localized states, from our finding we conclude that staggered stationary localized states vanish whenever $|\nu \lambda| \geq 1$. This happens due to an effectively repulsive on-site nonlinear potential. This potential helps spread the amplitude over the whole sample. We have already mentioned this. It is 


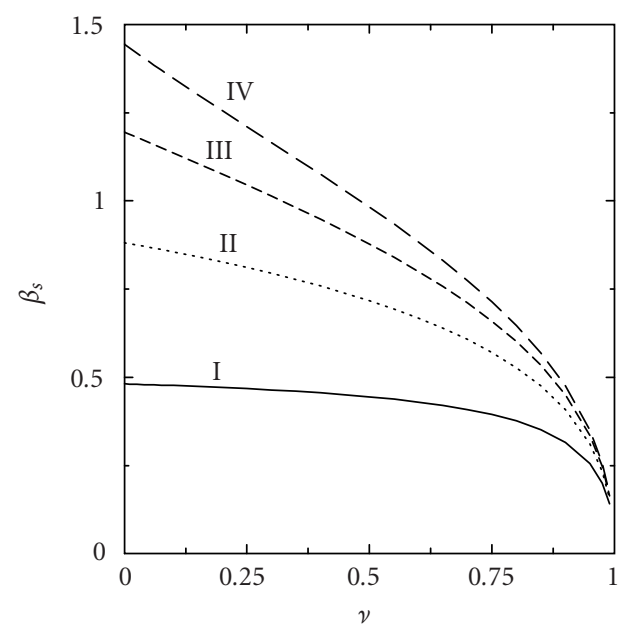

Figure 4.2. The variation of the smaller root $\beta_{s}$ of (4.3) in the text as a function of the nonintegrability parameter $\nu$ for various values of the parameter $a$. Since $\lambda=-1$, these states are staggered stationary localized states. Curve I: $a=0.5$, curve II: $a=1.0$, curve III: $a=1.5$, and curve IV: $a=2.0$.

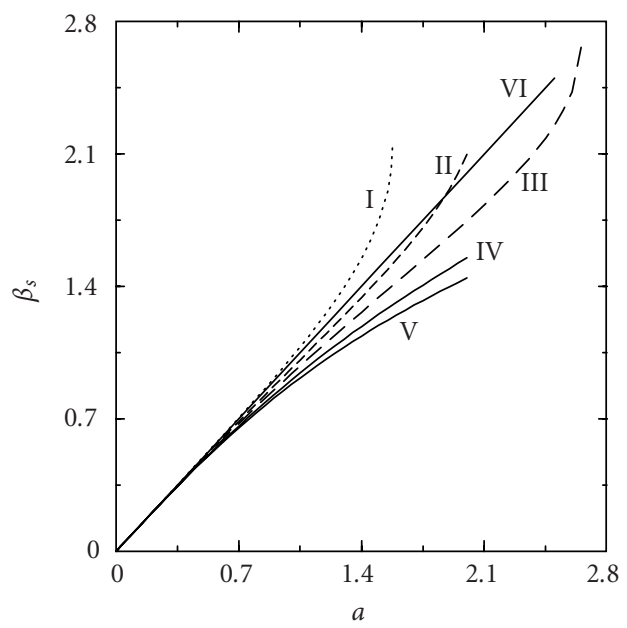

Figure 4.3. The variation of the smaller $\operatorname{root} \beta_{s}$ of (4.3) in the text as a function of the parameter $a$ for various values of the nonintegrability parameter $\nu$. Since $\lambda=1$, these states are unstaggered stationary localized states. Curve I: $v=0.75$, curve II: $\nu=0.40$, curve III: $v=0.25$, curve IV: $v=0.0$. Curve VI is the straight line, $\beta_{s}=a$.

also found for both unstaggered and staggered cases that for $a \sim o(1), a \sim \sinh \beta_{s} \sim \beta_{s}$, and hence, in this asymptotic limit, (4.1) together with (2.22) yields $\sqrt{\Psi} \sim \beta_{s} / \sqrt{1.0+v \lambda}$. Figure 4.3 shows the result for the unstaggered states with $v>0$. However, the result for staggered localized states is not shown here. This result agrees with the asymptotic result in [13]. 


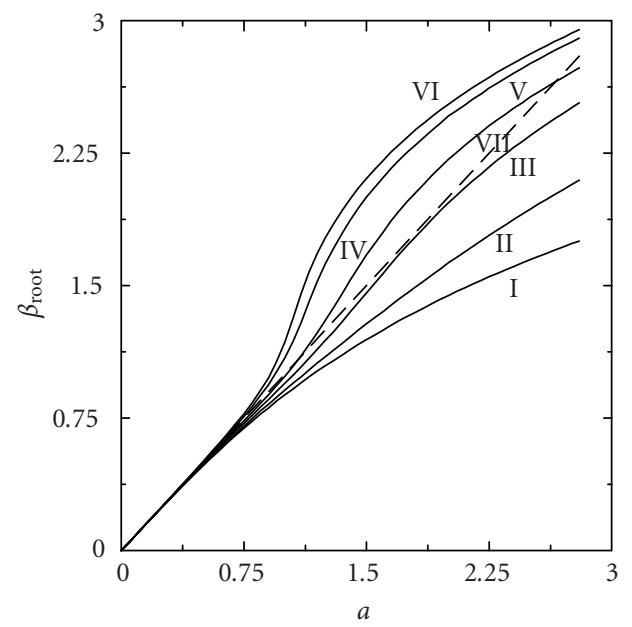

Figure 4.4. The variation of $\beta_{\text {root }}$ as a function of the parameter $a$, for various values of the nonintegrability parameter $\nu$. This is obtained from the exact calculation. For this figure, $\tilde{\mathrm{H}}_{0}=$ constant, $x_{0}=0.0$, and $\lambda=1$. Curve I: $\nu=0.0$, curve II: $\nu=0.10$, curve III: $\nu=0.40$, curve IV: $\nu=0.75$, curve V: $\nu=0.90$, and curve VI: $\nu=1.0$. Curve VII: the dashed curve is the straight line $\beta_{\text {root }}=a$.

The next important aspect is to study the effect of $x_{0}$ on the formation of these states. Another equally important aspect is to examine if unstable localized states that we find in the truncated equations or equivalently in the leading term analysis exist in the exact calculation. We first emphasize that the problem can be solved exactly in this reformulated version. For our numerical analysis, we use the "FindMinimum" program of Mathematica, version 4 . We discuss below the exact solution.

4.2. The exact solution. We consider first the case of unstaggered stationary localized states for $\nu>0$ and $x_{0}=0.0$. Figure 4.4 shows the variation of $\beta_{\text {root }}$ as a function of the parameter $a$ for various values of the nonintegrability parameter $\nu$. The corresponding figure to be compared is Figure 4.3. We note that for $\nu=0.0$, we obtain from (4.1) and (2.22) that $\Psi\left(\beta, 0.0, \lambda, x_{0}\right)=a \sinh \beta$. On the other hand, analytically $\Psi\left(\beta, 0.0, \lambda, x_{0}\right)=\sinh ^{2} \beta$. So, we must have then $a=\sinh \beta_{\text {root }}$. This is clearly obtained in our numerical analysis. As $\nu=0.0$ for these curves, both curve I of Figure 4.4 and curve $\mathrm{V}$ of Figure 4.3 are defined by the equation $\beta_{\text {root }}=\operatorname{arcsinh} a$. We further see in Figure 4.4 and also in Figure 4.3 that for small values of $a$, all curves merge simultaneously on the line $\beta_{\text {root }}=a$ and curve I (Figure 4.4) or curve V (Figure 4.3). This in turn implies that for $a \sim o(1), a \sim \sinh \beta_{\text {root }} \sim$ $\beta_{\text {root}}$. Hence, for on-site peaked unstaggered localized states, $\sqrt{\Psi} \sim \beta_{\text {root }} / \sqrt{1.0+\nu}$ is the asymptotic result [13]. We further note that in the exact calculation, we do not find any root corresponding to $\beta_{l}$ of (4.3) for any value of $a$. This conclusion is reached from the following observation in our numerical analysis. In our numerical analysis, we have used $N$ and $M$ number of terms in two infinite sums in (2.22) and (2.27), respectively. We find that $\beta_{l} \rightarrow \infty$ monotonically if both $N$ and $M \rightarrow \infty$, either separately or simultaneously. So, unstable stationary localized states obtained from (4.3) are spurious and due to the truncation error. Similar argument should hold good for the analysis of (3.10). 


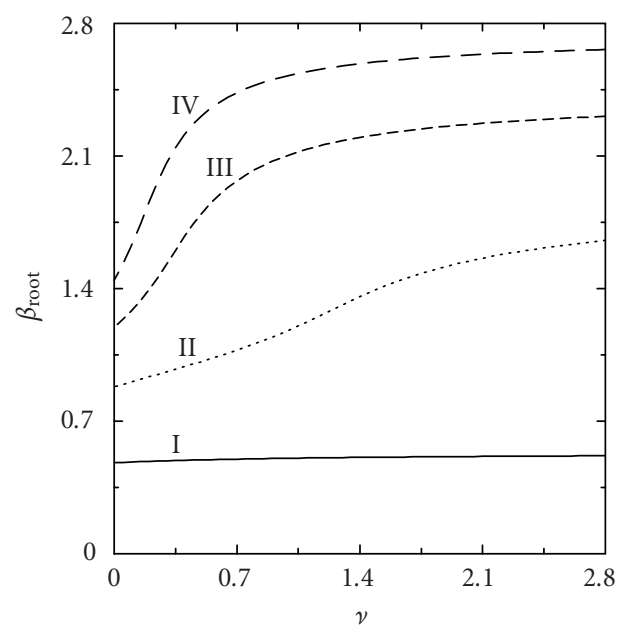

Figure 4.5. The variation of $\beta_{\text {root }}$ as a function of the nonintegrability parameter $\nu$ for various values of the parameter $a$. It is obtained from the exact calculation. For this figure, $\widetilde{\mathrm{H}}_{0}=$ constant, $x_{0}=0.0$, and $\lambda=1$. Curve I: $a=0.5$, curve II: $a=1.0$, curve III: $a=1.5$, and curve IV: $a=2.0$.

The variation of $\beta_{\text {root }}$ of unstaggered localized states as a function of $v$ for various values of $a$ from the exact calculation is shown in Figure 4.5.

We see that $\beta_{\text {root }}$ is a monotonically increasing function of $\nu$ for $v>0.0$. We note that $\beta_{\text {root }}^{-1}$ gives a measure of the width of the localized states. We have also argued before that the width of the stable unstaggered localized states for this case must decrease with increasing $\nu$. So, $\left|\beta_{\text {root }}\right|$ must increase with increasing $\nu$, if it were to characterize stable SLSs. Inasmuch as $\beta_{\text {root }}$ satisfies this criterion, stationary localized states corresponding to $\beta_{\text {root }}$ are stable.

For intersite peaked unstaggered localized states, having $x_{0}=0.5$, the dependence of $\beta_{\text {root }}$ on $a$ for a fixed $v$ is also investigated for various values of $v>0$. It is shown in Figure 4.6. Figure 4.6 also includes for comparison the variation of $\beta_{\text {root }}$ as a function of $a$ for $x_{0}=0.0$. We note that localized states have larger widths for $x_{0}=0.5$. This in turn implies that intersite peaked SLSs will have higher energy. Similarly, the variation of $\beta_{\text {root }}$ as a function of $\nu$ for $x_{0}=0.5$ is shown in Figure 4.7. We again note that stationary localized states for $x_{0}=0.5$ have larger widths. Most importantly intersite peaked SLSs show weak dependence on $v$. This is expected from physical considerations.

In Figure 4.8 we show the variation of energy of the on-site and intersite peaked stationary localized states as a function of $\nu$ for $x_{0}=0.0$ and 0.5 . When $v \sim o(1)$, the solution approximately has the continuous symmetry of the solution of $v=0.0[1,2,3]$. So, in this limit, both on-site and intersite peaked states should have almost the same energy. This is clearly seen in Figure 4.8. Similarly, on-site peaked states are supposed to be more stable than intersite peaked states. This is also clearly seen by comparing curves I(b) and II(b) in this figure. Again, when $a$ reduces, the width of the corresponding stationary localized state increases. Consequently, the energetic distinction between the on-site and intersite peaked states reduces. This is also clearly evident in Figure 4.8. 


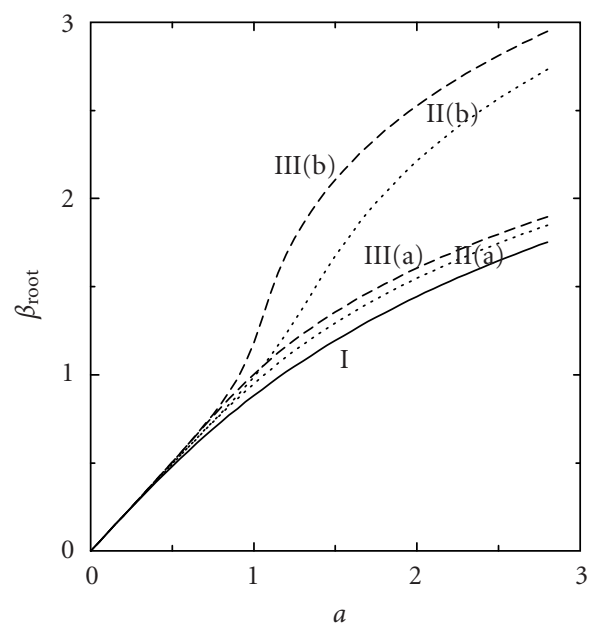

Figure 4.6. The variation of $\beta_{\text {root }}$ as a function of the parameter $a$ for two positive values of $\nu$. The exact solution for $\lambda=1$. For the curve I, $v=0.0$. For curves II(a) and II(b), $v=0.40$, but $x_{0}=0.5$ and 0.0 , respectively. For curves III(a) and III(b), $v=1.0$, but $x_{0}=0.5$ and 0.0 , respectively.

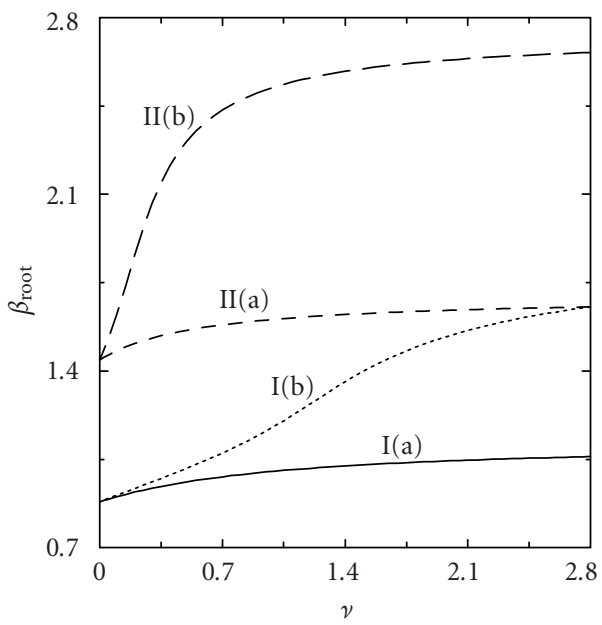

Figure 4.7. The variation of $\beta_{\text {root }}$ as a function of the nonintegrability parameter $v$ for two positive values of the parameter $a$. The exact solution for $\lambda=1$. For curves $\mathrm{I}(\mathrm{a})$ and $\mathrm{I}(\mathrm{b}), a=1$, but $x_{0}=0.5$ and 0.0 , respectively. For curves II(a) and II(b), $a=2.0$, but $x_{0}=0.5$ and 0.0 , respectively.

A comprehensive understanding of these results, delineating the basic differences of on-site peaked and intersite peaked unstaggered SLSs, is definitely required. To this end, we note that for $v>0$, these results indicate the operation of a nonlinear attractive potential in the system. This effective nonlinear potential is maximally attractive at lattice sites. From the physical consideration, we argue that the attractive potential assumes maximum 


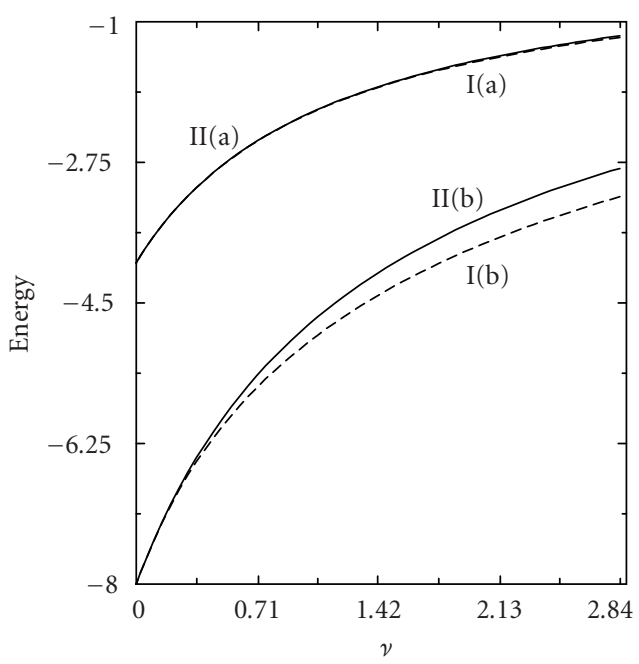

Figure 4.8. The variation of the energy of unstaggered stationary localized states as a function of $v$ for two values of $a$ and for two permissible values of $x_{0}$. Of course, the result is obtained from the exact calculation with $\tilde{\mathrm{H}}_{0}=$ constant. For curves $\mathrm{I}(\mathrm{a})$ and $\mathrm{II}(\mathrm{a}), a=1.0$, but $x_{0}=0.0$ and 0.5 , respectively. For curves I(b) and II(b), $a=2.0$, but $x_{0}=0.0$ and 0.5 , respectively.

values at the center of any two consecutive lattice sites. Since the system has lattice translational invariance, this potential will also have the periodicity of the underlying lattice. An attractive potential effectively reduces the intersite hopping of a particle, consequently helping its localization. Secondly, any state can be thought of as an effective particle with an effective mass, executing a motion in a potential. From the physical consideration, it is easy to see that the stronger an attractive potential is, the stronger the localization is. Consequently, the heavier the effective mass of the particle is. Conversely then the inverse of the effective mass gives the localization length of the effective state. In this picture then the unstaggered SLS for $v>0$ is equivalent to an effective particle sitting either at the bottom of any well $\left(x_{0}=0.0\right)$ or at the top of the same well $\left(x_{0}=0.5\right)$. So, the unstaggered SLS with $x_{0}=0.0$ will correspond to a heavier effective mass particle than the corresponding SLS with $x_{0}=0.5$. In terms of localization length, the first kind of states will be more localized than the second type. Another important deduction from this picture is that energetically the first kind of states should be more stable. These results are seen in our numerical analysis. Again, when we increase the parameter $a$, we increase the maximum amplitude of the SLS. In this effective picture, the depth of the potential well increases. A similar situation also occurs by increasing $\nu$. This in turn implies that the effective mass of the particle at the bottom of the well will increase with increasing $a$ and $\nu$. So, in both cases, the width of the on-site peaked unstaggered SLSs should decrease, as seen in our numerical calculation. The effective periodic potential however is a function of at least three variables, the position variable $x_{0}$, the parameter $a$, and the nonintegrability parameter $v$. Since the SLS with $x_{0}=0.5$ shows weak dependence on $v$, our results suggest that the top of the potential is not substantially affected by the change in $\nu$. On 


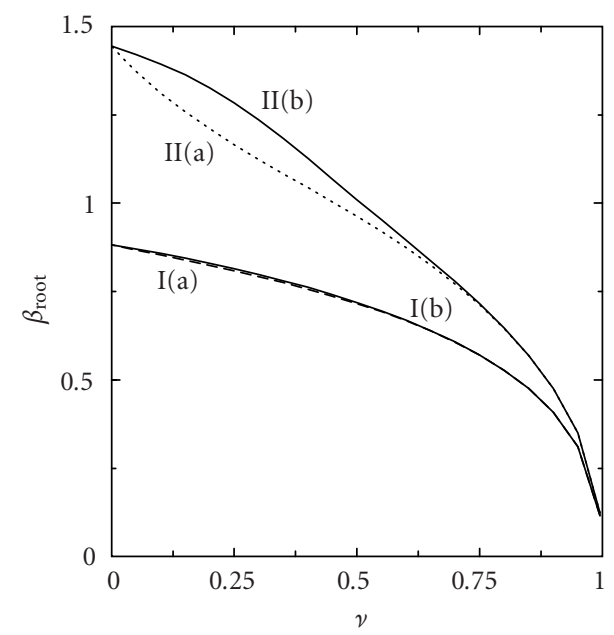

Figure 4.9. The variation of $\beta_{\text {root }}$ as a function of the nonintegrability parameter $v$ for $a=1.0$ and 2.0, as obtained from the exact calculation. Since $\lambda=-1$, these states are staggered stationary localized states. Curve I(a): $a=1.0$, and $x_{0}=0.0$. Curve II(a): $a=2.0$, and $x_{0}=0.0$. Curve I(b): $a=1.0$, and $x_{0}=0.5$. Curve II $(\mathrm{b}): a=2.0$, and $x_{0}=0.5$.

the other hand, from our results we deduce that the top of the potential is energetically stabilized by increase in $a$.

Finally, some exact calculations of $\beta_{\text {root }}$ for staggered SLSs for $\nu>0, \lambda=-1$ and for both $x_{0}=0.0$ and 0.5 are presented. In Figure 4.9 we present the variation of $\beta_{\text {root }}$ as a function of $v$ for $a=1.0$ and 2.0. Consider first $x_{0}=0.0$. Curves I(a) and II(a) in Figure 4.9 are almost identical to corresponding curves in Figure 4.2 with a discernible deviation in the magnitude of $\beta_{\text {root }}$ for large values of $a$ together with small values of $\nu$. The same calculation with $\tilde{\mathcal{N}}=$ constant gives $\beta_{\mathrm{s}} \rightarrow 0$ linearly as $\nu \lambda \rightarrow-1$. See Figure 3.5. Figure 4.10 shows the variation of $\beta_{\text {root }}$ as a function of $a$ for various values of $\nu>0$. The important point to note is that $\beta_{\text {root }}(\nu \lambda<0)<\beta_{\text {root }}(\nu=0)$. In other words, SLSs for $\nu>0$ are stabilized by expansion of the width. As $\beta_{\text {root }}=a$ line becomes tangent to all curves in Figure 4.10, we infer that when $a \sim o(1), \beta_{\text {root }} \rightarrow a$. Again on the curve $v=0, \sinh \beta_{\text {root }}=a$. In this asymptotic limit, we then have from (2.22) and (4.1) that $\sqrt{\Psi} \sim \beta_{\text {root }} / \sqrt{1-\nu}$. The corresponding approximate calculation for the model with $\tilde{\mathcal{N}}=$ constant is shown in Figure 3.6. Figure 3.6 shows that when $\alpha \sim o(1), \beta_{s} \sim \alpha^{2}(1-\nu)$. This in turn yields the same asymptotic result for $\sqrt{\Psi}$. Comparing our results for staggered SLSs from three different approaches, namely, approximate calculations with (a) $\widetilde{\mathcal{N}}=$ constant, (b) $\tilde{\mathrm{H}}_{0}=$ constant, and (c) the exact calculation with $\tilde{\mathrm{H}}_{0}=$ constant and $x_{0}=0.0$, we conclude that all three give qualitatively the same result.

We now consider the basic difference between staggered SLS with $x_{0}=0.0$ and staggered SLS with $x_{0}=0.5$. Figure 4.9 shows the variation of $\beta_{\text {root }}$ as a function of $\nu \geq 0$ for $a=1.0$ and 2.0. From this figure, we see that though $\beta_{\text {root }} \rightarrow 0$ as $\nu \rightarrow 1$ for both $x_{0}=0.0$ and 0.5 , the magnitude of $\beta_{\text {root }}$ for intermediate values of $\nu$ is greater for the SLSs with $x_{0}=0.5$. This in turn implies that the SLS with $x_{0}=0.5$ has smaller localization length. 


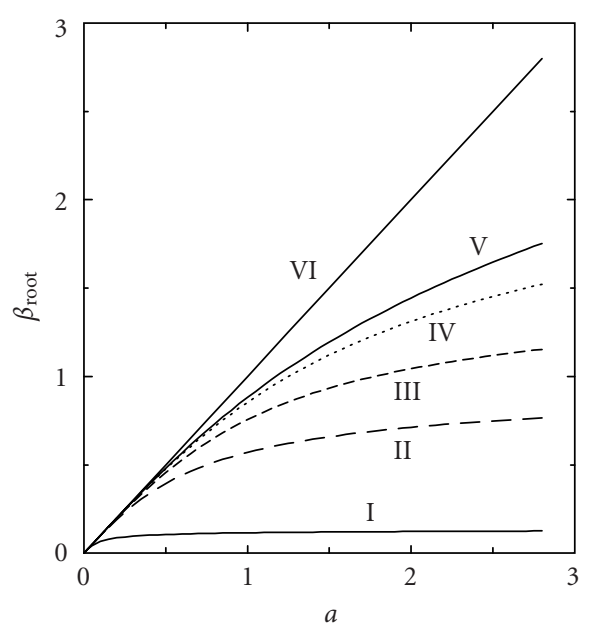

Figure 4.10. The variation of $\beta_{\text {root }}$ as a function of the parameter $a$ for positive values of $\nu$. The exact solution for $\lambda=-1$ and $x_{0}=0.0$. Curve I: $\nu=0.995$, curve II: $\nu=0.75$, curve III: $\nu=0.40$, curve IV: $\nu=0.1$, and curve $\mathrm{V}: \nu=0.0$. Curve VI: the solid curve is the straight line $\beta_{\text {root }}=a$.

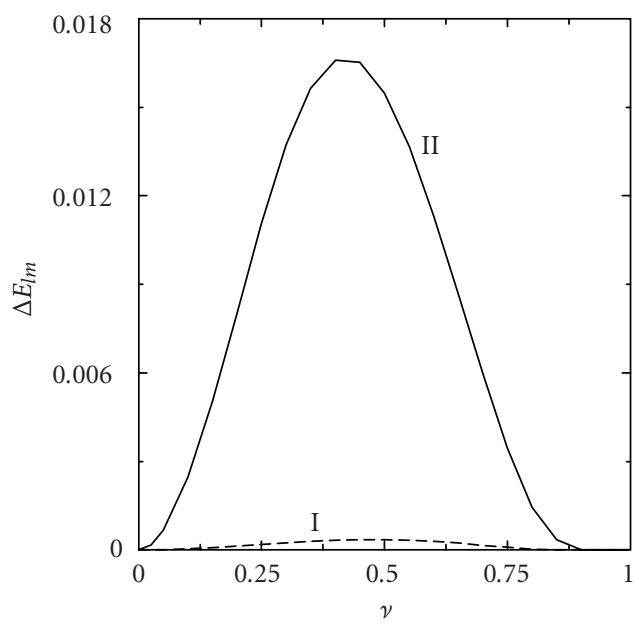

Figure 4.11. $\Delta E_{\mathrm{lm}}=E_{l}-E_{m}$, where $E_{l}$ and $E_{m}$ define the energy of staggered SLSs with $x_{0}=0.0$ and $x_{0}=0.5$, respectively. The variation of $\Delta E_{\mathrm{lm}}$ as a function for $v \in[0.1)$. Curve I: $a=1.0$ and curve II: $a=2.0$.

This is very much opposite to what we observe for an unstaggered SLS. We consider next Figure 4.11. We define $\Delta E_{\mathrm{lm}}=E_{l}-E_{m}$, where $E_{l}$ and $E_{m}$ define the energy of staggered SLS with $x_{0}=0.0$ and $x_{0}=0.5$, respectively. Figure 4.11 shows the variation of $\Delta E_{\mathrm{lm}}$ as a function for $\nu \in[0.1)$. As $\Delta E_{\mathrm{lm}}>0$ for intermediate values of $\nu$, the SLS with $x_{0}=0.5$ is energetically stable compared to its equal counterpart.

In further analysis, we note that the amplitude distribution of staggered SLS around the maximum amplitude site is ST-like mode for $x_{0}=0.0$ and P-like mode for $x_{0}=0.5$. 
In this case also there is a periodic arrangement of potential wells in the system. Since the on-site nonlinear potential is repulsive in this case, this potential will attain the minimum at the center of two consecutive lattice sites. So, the whole periodic arrangement of wells is shifted by half a lattice constant. Consequently, the effective particles corresponding to the P-like mode and the ST-like mode are sitting at the bottom of a well and at the top of a well, respectively [16]. So, the P-like mode corresponds to an effective particle with larger effective mass than the corresponding ST-like mode, and thereby having a smaller localization length. From this picture, we also deduce that the P-like mode is energetically more stable than the ST-like mode. This analysis further tells that ST-like mode will be unstable to infinitesimal perturbation, and the perturbation will cause it to move $[58,63,65]$. Most importantly however we prove by our variational approach that the existence of P-like mode and ST-like mode is a fundamental property of a system described by IN-DNLS. To successfully explain the dependence of the localization length of the P-like modes on these two parameters $a$ and $\nu$, respectively, we need the following behavior of the potential. When the parameter $a$ increases, the effective well depth must increase for intermediate values of $\nu$. Consequently, the effective mass of the particle will increase, and the localization length will decrease. But, in case of $\nu$, the effective well depth must decrease as $v \rightarrow 1$. Furthermore, as discussed in the context of unstaggered SLS, the dependence of the localization length of ST-like modes determines the dependence of the top of the potential well on two important parameters, $a$ and $\nu$.

\section{Summary}

IN-DNLS is a one-dimensional discrete nonlinear equation with a tunable nonintegrability parameter $v[13,62]$. When $v=0$, it reduces to the famous AL equations $[1,2,3]$. The importance of IN-DNLS in physics as well as in nonlinear mathematics is discussed in the text. In this paper, primarily eigenvalues, energies, and corresponding site amplitudes of SLSs of IN-DNLS are studied using discrete variational formulation [5, 32, 48, 51]. The standard variational approach starts from the respective Lagrangian to study this type of problem. In this paper, however, the appropriate functional is derived using the standard variational procedure for finding eigenvalues of Sturm-Liouville equations [33]. In other words, it is shown here how the effective functional can be derived from the Hamiltonian and constants of motion without the prior knowledge of the Lagrangian. The uniqueness of the functional is also established by showing its equivalence to the effective Lagrangian.

Inasmuch as localized states in one-dimensional linear impure as well as disordered systems show asymptotic exponential decay $[22,36,57]$, a "sech" ansatz with two parameters, $\beta$ and $\Phi=\sqrt{\Psi}$, is used to find eigenvalues and eigenfunctions. In this choice, $\beta^{-1}$ and $\sqrt{\Psi}$ define the width and the maximum amplitude of SLS, respectively. This ansatz is so chosen as it gives AL stationary localized states when $v \rightarrow 0$. Furthermore, SLSs of IN-DNLS are assumed to belong to the class of breathers with a single frequency [26]. Since stationary solitons of AL equations are breathers of this class, this choice of form for SLSs of IN-DNLS is justified.

Very naturally two procedures have emanated in our variational calculation. In the first case, the reduced dynamical system is described by the Hamiltonian $\tilde{H}_{0}$ and $\tilde{\mathcal{N}}$ is taken to be the number constant. In the second case, $\tilde{\mathcal{N}}$ acts as the Hamiltonian. Since 
the analysis involves two infinite sums, both sums are ignored in both cases in the leading term analysis. In both cases, for unstaggered stationary localized states two permissible values, namely, $\beta_{s}$ and $\beta_{l}$, of the width parameter $\beta$ are found. It is further found that for two real roots to exist we need $v<v_{\text {critical }}$ if $v>0$. Furthermore, $v_{\text {critical }}$ is found to be a monotonically decreasing function of the parameter $\alpha$ in the first case and $a$ in the second case. These parameters are defined in the text and are positive semidefinite. It is successfully argued from our numerical analysis that SLSs, characterized by the smaller width parameter $\beta_{s}$, are stable and the states characterized by $\beta_{l}$ are unstable. However, for staggered SLSs both procedures have yielded a single value, $\beta_{s}$, and our numerical results indicate that these are stable localized modes of the system. Though both procedures yield qualitatively, the same results for the parameters of SLSs, no quantitative comparison is attempted here. Again, it is found that the problem can be exactly solved in the second case. In our exact solution no unstable SLS is obtained. So, the occurrence of unstable SLSs in this system in the leading term analysis should be attributed to the truncation error. In the context of SLS in one-dimensional nonlinear systems, this is indeed an important result.

The formation of unstaggered and staggered SLSs are investigated here for $v \geq 0$. For the null value of $\nu$, the present variational procedure correctly produces SLSs of AL equation. Furthermore, when $\nu \rightarrow 0$, it is found that $\beta_{s} \rightarrow \alpha^{2}$ in the first case for both unstaggered and staggered SLSs. Consequently, AL stationary soliton is recovered in this asymptotic limit. In the second case, $a \rightarrow \sinh \beta_{s}$ asymptotically as $\nu \rightarrow 0$ in the leading term analysis. This result is true for both unstaggered and staggered SLSs. The same asymptotic results are found in the exact analysis too, except that $\beta_{s}$ is replaced by $\beta_{\text {root }}$. So, in the second case too the AL stationary soliton is the asymptotic result for $\nu \rightarrow 0$. Analytically also the same asymptotic result is obtained here. Our both analytical and numerical results are expected on physical consideration.

In the other asymptotic analysis, $\alpha \rightarrow 0$ in the first case and $a \rightarrow 0$ in the second case. Again, in the second case there are two scenarios, the leading term analysis and the exact calculation. For all cases and for both unstaggered and staggered localized states, the known asymptotic form of $\Psi$ for the stable SLS are obtained numerically from the present variational analysis. This is therefore a very important contribution of the present work. For unstaggered SLSs, it is found that the width of the state decreases with increasing $\nu$. On the other hand, for staggered SLSs, the width increases with increasing $v$ and vanishes as $\nu \rightarrow 1$. These results are consistent with the physics of the problem and reasons are given in the text.

Another important aspect is the dependence of the width of SLSs on the position of the maximum amplitude, denoted by $x_{0}$. It is proved in the text that $x_{0}=0$ or $\pm 1 / 2$. It is observed in our analysis that for unstaggered SLSs, the on-site peaked SLS $\left(x_{0}=0\right)$ has smaller width than the intersite peaked $\left(x_{0}= \pm 1 / 2\right)$ SLS for a given value $\nu>0$ and $a>0$. Our analysis also shows that for a given $v$ and $a$, the on-site peaked unstaggered SLS is energetically more stable than the corresponding intersite peaked SLS. These results are physically realistic and are successfully explained using the effective-mass picture. It is found in our analysis that the existence of the P-like mode and the ST-like mode is a fundamental property of the system described by IN-DNLS. It is also shown in this 
numeroanalytical method that the P-like mode is energetically more stable than the corresponding ST-like mode. These results constitute a very important contribution of the present work.

It is definitely important to find exact eigenvalues and eigenvectors of the problem. Present analysis may turn out to be a useful guide for the exact calculation. In this analysis we have not proved that the lowest eigenvalue is obtained. Furthermore, the system may have more than one SLS type of ILMs. These questions need to be properly investigated. Presence of impurity in the nonintegrability parameter $v$ may produce more stationary localized states and these states may interact through further external perturbation. A study of this type is important in the transport in nonlinear systems [7, 44]. In our calculation, we find both P-like mode and ST-like mode. It will be interesting to know the asymptotic form of these modes in this model. Furthermore, the behavior of these modes under external perturbation should also be investigated. Finally, it is important to find more physical as well as biological systems, where IN-DNLS can be used to study transport properties. A good candidate in this regard is the transport across biological membranes of protons through proton wires.

\section{Appendices}

\section{A. The formulation of discrete variational approach for IN-DNLS}

We are dealing with a nonlinear eigenvalue problem in our aim to find stationary localized states of IN-DNLS equations, (2.4) and (2.5) in the text. For this purpose we are employing variational formulation $[5,6,33]$. To implement the variational approach for this problem, we require the proper functional $\widetilde{\mathrm{F}}$ whose constrained variation will lead to $(2.5)$ [33]. We of course know a constant of motion and the Hamiltonian, $\tilde{\mathcal{N}}$ and $\tilde{\mathrm{H}}$, respectively for the problem at hand [13].

Inasmuch as we know $\widetilde{\mathcal{N}}$ and $\tilde{\mathrm{H}}$, using the analogous variational approach of finding eigenvalues in standard Sturm-Liouville problems [33]. We set up the functional $\widetilde{\mathrm{F}}=$ $\tilde{\mathrm{H}}-\Lambda \tilde{\mathcal{N}}$ where $\Lambda$ is the Lagrange multiplier [35]. We then have for the variation of $\widetilde{\mathrm{F}}$, $\delta \tilde{\mathrm{F}}=\delta \tilde{\mathrm{H}}-\Lambda \delta \tilde{\mathcal{N}}$. For the calculation of the variation, we transform $\Psi_{n} \rightarrow \Psi_{n}+\delta \Psi_{n}, n \in$ $Z$, in the expression of $\tilde{\mathrm{H}}$ and $\tilde{\mathcal{N}}((2.6)$ and $(2.7)$, resp.) to obtain

$$
\delta \widetilde{\mathrm{F}}=\delta \tilde{\mathrm{H}}-\Lambda \delta \tilde{\mathcal{N}}=-2 \sum_{n} \frac{\lambda\left(1+\Psi_{n}^{2}\right)\left(\Psi_{n+1}+\Psi_{n-1}\right)+2 \nu \Psi_{n}^{3}+\Lambda \Psi_{n}}{1+\Psi_{n}^{2}} \delta \Psi_{n}
$$

Since $\left\{\delta \Psi_{n}\right\}$ are arbitrary, $\delta \widetilde{\mathrm{F}}=0$ implies that

$$
\lambda\left(1+\Psi_{n}^{2}\right)\left(\Psi_{n+1}+\Psi_{n-1}\right)+2 \nu \Psi_{n}^{3}+\Lambda \Psi_{n}=0
$$

We note that (A.2) is identical to (2.5) when $\Lambda=\omega$. From further analysis, we find that $\omega$ is given by (2.11) in the text.

For the case where $\tilde{\mathrm{H}}_{0}=$ constant, the corresponding functional $\widetilde{\mathrm{F}}$ should be given by $\widetilde{\mathrm{F}}=\Lambda_{2} \tilde{\mathrm{H}}_{0}+2 \nu \tilde{\mathcal{N}}$, where $\left(\Lambda_{2}-1\right)$ is the Lagrange multiplier. The same procedure will yield (2.5) if $\Lambda_{2}=2 v /(2 v-\omega)$. 
So, it is important to note that we can devise the required functional to determine the eigenvalues of SLSs by variational approach without the formal knowledge of the Lagrangian.

\section{B. Calculation of the function $\tilde{\mathcal{N}}\left(\Psi, \beta, x_{0}\right),(2.27)$}

Before we proceed in this section, we cite some results required for the calculation $[4,28]$ :

$$
I(s, \Psi, \beta)=\int_{0}^{\infty} d y \frac{\cos (\pi s / \beta) y}{\cosh y+(1+2 \Psi)}=\frac{\pi \sin \{(2 \pi s / \beta) \operatorname{arcsinh} \sqrt{\Psi}\}}{2 \sqrt{\Psi(1+\Psi)} \sinh \left(\pi^{2} s / \beta\right)} .
$$

From (B.1) we get

$$
\lim _{s \rightarrow 0} I(s, \Psi, \beta)=\frac{\operatorname{arcsinh} \sqrt{\Psi}}{\sqrt{\Psi}(1+\Psi)}=\frac{d(\operatorname{arcsinh} \sqrt{\Psi})^{2}}{d \Psi} .
$$

In our calculation, we are using the following ansatz:

$$
\Psi_{n}=\Phi \frac{1}{\cosh \beta\left(n-x_{0}\right)}, \quad n \in Z
$$

This ansatz has also been used in the previous analysis [13]. For on-site peaked and STlike localized states, $x_{0}=0$, and for intersite peaked and P-like states, $x_{0}= \pm 1 / 2[5,58,63$, 65]. We further write $\Phi^{2}=\Psi$. The function $\tilde{\mathcal{N}}\left(\Psi, \beta, x_{0}\right)$ is given by $(2.27)$ in the text. Now introducing (B.3) in (2.6) and then taking partial derivative with respect to $\Psi$, we get

$$
\frac{\partial \tilde{\mathcal{N}}}{\partial \Psi}=\sum_{n=-\infty}^{\infty} \frac{1}{\cosh ^{2} \beta\left(n-x_{0}\right)+\Psi}
$$

We use next the famous Poisson sum formula (2.17) in the text in (B.4) [72]. Thereafter, some simple algebraic manipulations are done to obtain

$$
\frac{\partial \tilde{\mathcal{N}}}{\partial \Psi}=\frac{2}{\beta} I(0, \Psi, \beta)+\frac{4}{\beta} \sum_{s=1}^{\infty} \cos \left(2 \pi s x_{0}\right) I(s, \Psi, \beta)
$$

We note that (B.5) is identical to (2.20) in the text. Furthermore, we have from the definition that $\tilde{\mathcal{N}}\left(0, \beta, x_{0}\right)=0$. See (2.6) in the text. After integration of $(\mathrm{B} .5)$ over $\Psi^{\prime} \in(0, \Psi)$ we get (2.27) in the text. When $\Psi=\sinh ^{2} n \beta, n \in Z$, in (2.27), we get $\tilde{\mathcal{N}}\left(\Psi, \beta, x_{0}\right)=2 n^{2} \beta$. See (3.1) in this context. This particular result has been obtained by another route in the literature $[10,12]$. We consider now $v=0$ or the AL equation. Then from $(2.12),(2.20)$, (2.25), (2.26), and (2.28), we get for $n \in N$,

$$
\tanh n \beta \operatorname{coth} \beta-n=0
$$

We see then that if $n>1,($ B.6) has no real nonzero $\beta$ as a solution. 


\section{Application of the method of successive substitutions in the investigation of the for- mation of stationary localized states in IN-DNLS}

Following the text, we take $\tilde{\mathcal{N}}\left(\Psi, \beta, x_{0}\right)=2 \alpha^{2}=$ Constant. We further write $(2.27)$ in the form $\Psi=F(\Psi)$ where the function $F(\Psi)$ is defined as

$$
F(\Psi)=\sinh ^{2}\left[\sqrt{\alpha^{2} \beta-f_{2}\left(\beta, x_{0}, \Psi\right)}\right]
$$

and in (C.1)

$$
f_{2}\left(\beta, x_{0}, \Psi\right)=2 \beta \cos \left[2 \pi x_{0}\right] \frac{\sin ^{2}((\pi / \beta) \operatorname{arcsinh} \sqrt{\Psi})}{\sinh \left(\pi^{2} / \beta\right)} .
$$

It should be noted that only the first term in the sum in (2.27) is retained to obtain (C.1). To obtain roots of the equation $\Psi=F(\Psi)$, we can use the method of successive substitutions. In this method at the $k$ th iteration, we write $\Psi_{k+1}=F\left(\Psi_{k}\right)$ with the assumption that $\lim _{k \rightarrow \infty} \Psi_{k} \rightarrow \Psi_{\text {root }}$. However, the necessary condition for this to happen is that $\left|F^{\prime}\left(\Psi_{\text {root }}\right)\right|<1$ [34]. To explain the use of this method in the calculation, we will restrict ourselves only to the first iteration with $\Psi_{0}=\sinh ^{2} \alpha \sqrt{\beta}$. This in turn implies that

$$
\Psi \sim \Psi_{1}=\sinh ^{2}\left[\sqrt{\alpha^{2} \beta-f_{2}\left(\beta, x_{0}, \Psi_{0}\right)}\right] .
$$

Note that in the calculation of roots $((3.10)$ in the text $)$ we have taken $f_{2}=0$. By approximating $\Psi$ by $\Psi_{1}$ and furthermore keeping only the first term in the sum in the definition of $f_{1}\left(\beta, \nu, \lambda, x_{0}\right)(2.22)$ we get from (2.24)

$$
\tilde{\mathrm{H}}_{0}=-4 \lambda \frac{\Psi_{1}}{\sinh \beta}-4 \lambda \nu \frac{\Psi_{1}}{\beta}\left[1+2 \cos 2 \pi x_{0} \frac{\pi^{2} / \beta}{\sinh \left(\pi^{2} / \beta\right)}\right] .
$$

We then get the permissible values of $\beta$ by setting $d \tilde{\mathrm{H}}_{0} / d \beta=0$.

\section{References}

[1] M. J. Ablowitz and P. A. Clarkson, Solitons, Nonlinear Evolution Equations and Inverse Scattering, London Mathematical Society Lecture Note Series, vol. 149, Cambridge University Press, Cambridge, 1991.

[2] M. J. Ablowitz and J. F. Ladik, Nonlinear differential-difference equations, J. Mathematical Phys. 16 (1975), 598-603.

[3] _ Nonlinear differential-difference equations and Fourier analysis, J. Mathematical Phys. 17 (1976), no. 6, 1011-1018.

[4] M. Abramowitz and I. A. Stegun, Handbook of Mathematical Functions, 9th ed., Dover Publications, New York, 1970.

[5] A. B. Aceves, C. De Angelis, T. Peschel, et al., Discrete self-trapping, soliton interactions, and beam steering in nonlinear waveguide arrays, Phys. Rev. E (3) 53 (1996), no. 1, 1172-1189.

[6] D. Anderson, Variational approach to pulse propagation in optical fibers, Phys. Rev. A 27 (1983), no. 6, 3135-3145.

[7] S. Aubry and T. Cretegny, Mobility and reactivity of discrete breathers, Phys. D 119 (1998), no. 1$2,34-46$. 
[8] O. Bang and M. Peyrard, Generation of high-energy localized vibrational modes in nonlinear Klein-Gordon lattices, Phys. Rev. E (3) 53 (1996), no. 4, 4143-4152.

[9] O. M. Braun and Y. S. Kivshar, Nonlinear dynamics of the Frenkel-Kontorova model, Phys. Rep. 306 (1998), no. 1-2, 108.

[10] D. A. Cai, A. R. Bishop, and N. Grønbech-Jensen, Spatially localized, temporally quasiperiodic, discrete nonlinear excitations, Phys. Rev. E (3) 52 (1995), no. 6, R5784-R5787.

[11] _ Discrete lattice effects on breathers in a spatially linear potential, Phys. Rev. E (3) 53 (1996), no. 1, 1202-1205.

[12] - Perturbation theories of a discrete, integrable nonlinear Schrödinger equation, Phys. Rev. E (3) 53 (1996), no. 4, part B, 4131-4136.

[13] D. A. Cai, A. R. Bishop, and N. GrønbechJensen, Localized states in discrete nonlinear Schrödinger equations, Phys. Rev. Lett. 72 (1994), no. 5, 591-595.

[14] A. Campa and A. Giasanti, Experimental test for the Peyrard-Bishop model applied to the melting of very short DNA chains, Phys. Rev. E (3) 58 (1998), no. 3, 3585-3588.

[15] P. M. Chaikin and T. C. Lubensky, Principle of Condensed Matter Physics, Cambridge Press University, Cambridge, 1998.

[16] C. Claude, Y. S. Kivshar, O. Kluth, and K. H. Spatschek, Moving localized modes in nonlinear lattices, Phys. Rev. B 47 (1993), no. 21, 14228-14232.

[17] S. Darmanyan, A. Kobyakov, and F. Lederer, Stability of strongly localized excitations in discrete media with cubic nonlinearity, Ž. Èksper. Teoret. Fiz. 113 (1998), no. 4, 1253-1260, translated in JETP Lett. 86 (1998), no. 4, 682-686.

[18] A. Das, Integrable Models, World Scientific Lecture Notes in Physics, vol. 30, World Scientific Publishing, New Jersey, 1989.

[19] S. V. Dmitriev, Y. S. Kivshar, and T. Shigenari, Fractal structures and multiparticle effects in soliton scattering, Phys. Rev. E (3) 64 (2001), no. 5, 056613-056614.

[20] P. G. Drazin and R. S. Johnson, Solitons: An Introduction, Cambridge Texts in Applied Mathematics, Cambridge University Press, Cambridge, 1989.

[21] D. H. Dunlap, H.-L. Wu, and P. Phillips, Absence of localization in a random-dimer model, Phys. Rev. Lett. 65 (1990), no. 1, 88-91.

[22] E. N. Economou, Green's Functions in Quantum Physics, Springer Series in Solid-State Sciences, vol. 7, Springer, Berlin, 1983.

[23] H. S. Eisenberg, Y. Silberberg, R. Morandotti, A. R. Boyd, and J. S. Aitchison, Discrete spatial optical solitons in waveguide arrays, Phys. Rev. Lett. 81 (1998), no. 16, 3383-3386.

[24] S. Flach, K. Kladko, and R. S. MacKay, Energy thresholds for discrete breathers in one-, two- and three-dimensional lattices, Phys. Rev. Lett. 78 (1997), no. 7, 1207-1210.

[25] S. Flach and R. S. MacKay (eds.), A special issue on discrete breathers, Phys. D 119 (1998), 1-238.

[26] S. Flach and C. R. Willis, Discrete breathers, Phys. Rep. 295 (1998), no. 5, 181-264.

[27] A. Ghosh, B. C. Gupta, and K. Kundu, Stationary self-localized states due to quadratic nonlinearity in one-dimensional systems, J. Phys.: Condens. Matter 10 (1998), no. 12, 2701-2713.

[28] I. S. Gradshteyn and I. M. Ryzhik, Table of Integrals, Series, and Products, 4th ed., Academic Press [Harcourt Brace Jovanovich Publishers], New York, 1980.

[29] B. C. Gupta and K. Kundu, Formation of stationary localized states due to nonlinear impurities using the discrete nonlinear Schrödinger equation, Phys. Rev. B 55 (1997), no. 2, 894-905.

[30] _ Localized states in 1-D nonlinear chain, Phys. Lett. A 235 (1997), 176-182.

[31] Stationary localized states due to a nonlinear dimeric impurity embedded in a perfect one dimensional chain, Phys. Rev. B 55 (1997), no. 17, 11033-11036.

[32] _ Discrete nonlinear Schrödinger equation and stationary localized states, Nonlinear Dynamics: Integrability and Chaos (M. Daniel, K. M. Tamizhmani, and R. Sahadevan, eds.), Narosa publishing House, New Delhi, 2000, pp. 193-199.

[33] F. B. Hildebrand, Methods of Applied Mathematics, 2nd ed., Prentice-Hall, New Jersey, 1965. 


\section{A discrete variational approach for investigation in IN-DNLS}

[34] Introduction to Numerical Analysis, 2nd ed., International Series in Pure and Applied Mathematics, McGraw-Hill Book, New York, 1974.

[35] - Advanced Calculus for Applications, 2nd ed., Prentice-Hall, New Jersey, 1976.

[36] K. Ishii, Localization of eigenstates and transport phenomena in the one-dimensional disordered system, Theoret. Phys. Suppl. 53 (1973), 77-138.

[37] M. Johansson and Y. S. Kivshar, Discreteness-induced oscillatory instabilities of dark solitons, Phys. Rev. Lett. 82 (1999), no. 1, 85-88.

[38] D. J. Kaup and T. I. Lakoba, Variational method: how it can generate false instabilities, J. Math. Phys. 37 (1996), no. 7, 3442-3462.

[39] P. G. Kevrekidis, A. R. Bishop, and K. Ø. Rasmussen, Twisted localized modes, Phys. Rev. E 63 (2001), no. 3, 036603.

[40] J. M. Khalack, Y. Zolotaryuk, and P. L. Christiansen, Discrete breathers in classical ferromagnetic lattices with easy-plane anisotropy, Chaos 13 (2003), no. 2, 683-692.

[41] Y. S. Kivshar and D. K. Campbell, Peierls-Nabarro potential barrier for highly localized nonlinear modes, Phys. Rev. E (3) 48 (1993), no. 4, 3077-3081.

[42] Y. S. Kivshar and M. Peyrard, Modulational instabilities in discrete lattices, Phys. Rev. A (3) 46 (1992), no. 6, 3198-3205.

[43] Y. S. Kivshar, F. Zhang, and A. S. Kovalev, Stable nonlinear heavy-mass impurity modes, Phys. Rev. B 55 (1997), no. 21, 14265-14269.

[44] G. Kopidakis, S. Aubry, and G. P. Tsironis, Targeted energy transfer through discrete breathers in nonlinear systems, Phys. Rev. Lett. 87 (2001), no. 16, 165501.

[45] K. Kundu, Perturbative study of classical Ablowitz-Ladik type soliton dynamics in relation to energy transport in $\alpha$-helical proteins, Phys. Rev. E (3) 61 (2000), no. 5, part B, 5839-5851.

[46] - A study of a new class of discrete nonlinear Schrödinger equations, J. Phys. A 35 (2002), no. 38, 8109-8133.

[47] K. Kundu, D. Giri, and K. Ray, A study of one-dimensional correlated disordered systems using the invariant measure method, J. Phys. A 29 (1996), no. 17, 5699-5717.

[48] K. Kundu and B. C. Gupta, The role of power law nonlinearity in the discrete nonlinear Schrödinger equation on the formation of stationary localized states in the Cayley tree, Eur. Phys. J. B Condens. Matter Phys. 3 (1998), no. 1, 23-33.

[49] J. Kutz, C. Hile, W. Kath, R.-D. Li, and P. Kumar, Pulse propagation in nonlinear optical fiber lines that employ phase-sensitive parametric amplifiers, J. Opt. Soc. Amer. B Opt. Phys. 11 (1994), no. 10, 2112-2123.

[50] R. S. MacKay and S. Aubry, Proof of existence of breathers for time-reversible or Hamiltonian networks of weakly coupled oscillators, Nonlinearity 7 (1994), no. 6, 1623-1643.

[51] B. Malomed and M. I. Weinstein, Soliton dynamics in the discrete nonlinear Schrödinger equation, Phys. Lett. A 220 (1996), no. 1-3, 91-96.

[52] B. A. Malomed and R. S. Tasgal, Vibration modes of a gap soliton in a nonlinear optical medium, Phys. Rev. E (3) 49 (1994), no. 6, 5787-5796.

[53] B. A. Malomed and J. Yang, Solitons in coupled Ablowitz-Ladik chains, Phys. Lett. A 302 (2002), no. $4,163-170$.

[54] J. L. Marín and S. Aubry, Breathers in nonlinear lattices: numerical calculation from the anticontinuous limit, Nonlinearity 9 (1996), no. 6, 1501-1528.

[55] J. L. Marín, S. Aubry, and L. M. Floría, Intrinsic localised modes. Existence and linear stability, Phys. D 113 (1998), no. 2-4, 283-292.

[56] P. Marquié, J. M. Bilbault, and M. Remoissenet, Observation of nonlinear localized modes in an electrical lattice, Phys. Rev. E (3) 51 (1995), no. 6, 6127-6133.

[57] H. Matsuda and K. Ishii, Localization of normal modes and energy transport in the disordered harmonic chain, Progr. Theoret. Phys. (1970), no. Suppl. 45, 56-86. 
[58] J. B. Page, Asymptotic solutions for localized vibrational modes in strongly anharmonic periodic systems, Phys. Rev. B 41 (1990), no. 11, 7835-7838.

[59] M. Peyrard and A. R. Bishop, Statistical mechanics of a nonlinear model for DNA denaturation, Phys. Rev. Lett. 62 (1989), no. 23, 2755-2758.

[60] K. Ø. Rasmussen, S. Aubry, A. R. Bishop, and G. P. Tsironis, Discrete nonlinear Schrödinger breathers in a phonon bath, Eur. Phys. J. B Condens. Matter Phys. 15 (2000), no. 1, 169-175.

[61] R. Z. Sagdeev, S. S. Moiseev, A. V. Tur, and V. V. Yanevskii, Problems of the theory of string turbulence and topological solitons, Nonlinear Phenomena in Plasma Physics and Hydrodynamics (R. Z. Sagdeev, ed.), Mir Publishers, Moscow, 1986, p. 137.

[62] M. Salerno, Quantum deformations of the discrete nonlinear Schrödinger equation, Phys. Rev. A (3) 46 (1992), no. 11, 6856-6859.

[63] K. W. Sandusky, J. B. Page, and K. E. Schmidt, Stability and motion of intrinsic localized modes in nonlinear periodic lattices, Phys. Rev. B 46 (1992), no. 10, 6161-6168.

[64] A. Scott, Nonlinear Science. Emergence and Dynamics of Coherent Structures, Oxford Texts in Applied and Engineering Mathematics, vol. 1, Oxford University Press, Oxford, 1999.

[65] A. J. Sievers and S. T. Takeno, Intrinsic localized modes in anharmonic crystals, Phys. Rev. Lett. 61 (1988), no. 8, 970-973.

[66] B. I. Swanson, J. A. Brozik, S. P. Love, G. F. Strouse, and A. P. Shreve, Observation of intrinsically localized modes in a discrete low-dimensional material, Phys. Rev. Lett. 82 (1999), no. 16, 3288-3291.

[67] S. Takeno, Exact anharmonic-localized-mode solutions to the d-dimensional discrete nonlinear Schrödinger equation, J. Phys. Soc. Japan 58 (1989), no. 3, 759-762.

[68] A. Trombettoni and A. Smerzi, Discrete solitons and breathers with dilute bose-einstein condensates, Phys. Rev. Lett. 86 (2001), no. 11, 2353-2356.

[69] G. P. Tsironis and S. Aubry, Slow relaxation phenomena induced by breathers in nonlinear lattices, Phys. Rev. Lett. 77 (1996), no. 26, 5225-5228.

[70] A. A. Vakhnenko and Y. B. Gaĭdideŭ, The character of the motion of solitons in discrete molecular chains, Teoret. Mat. Fiz. 68 (1986), no. 3, 350-359 (Russian), Theor. Math. Phys. 68 (1987), 873-880.

[71] V. E. Zakharov and A. B. Shabat, Exact theory of two-dimensional self-focusing and onedimensional self-modulation of waves in nonlinear media, Ž. Èksper. Teoret. Fiz. 61 (1971), no. 1, 118-134, translated in Soviet Phys. JET 34 (1972), no. 1, 62-69.

[72] J. M. Ziman, Principles of the Theory of Solids, 2nd ed., Cambridge University Press, London, 1972.

K. Kundu: Institute of Physics, Bhubaneswar 751005, Orissa, India

E-mail address: kundu@iopb.res.in 


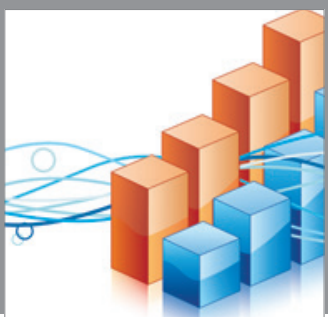

Advances in

Operations Research

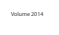

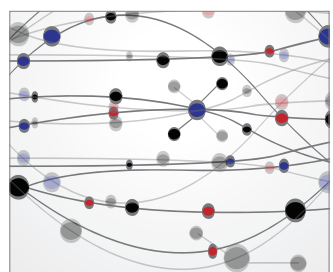

\section{The Scientific} World Journal
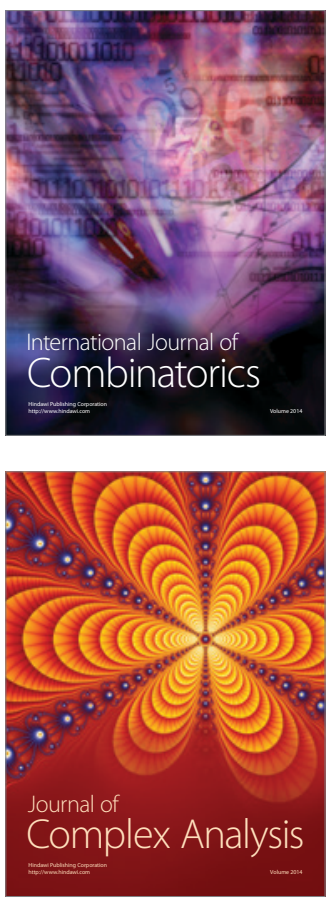

International Journal of

Mathematics and

Mathematical

Sciences
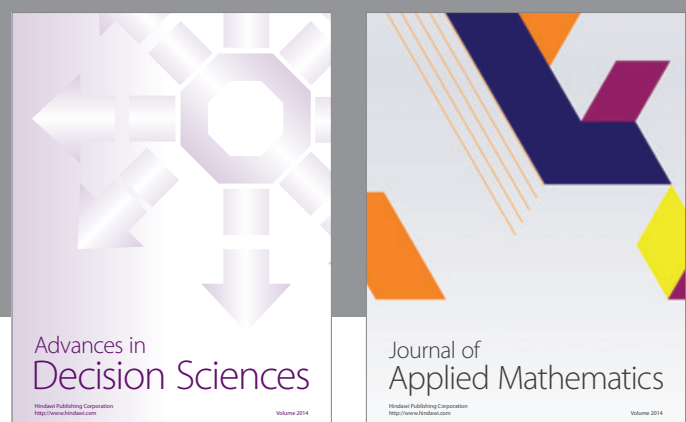

Journal of

Applied Mathematics
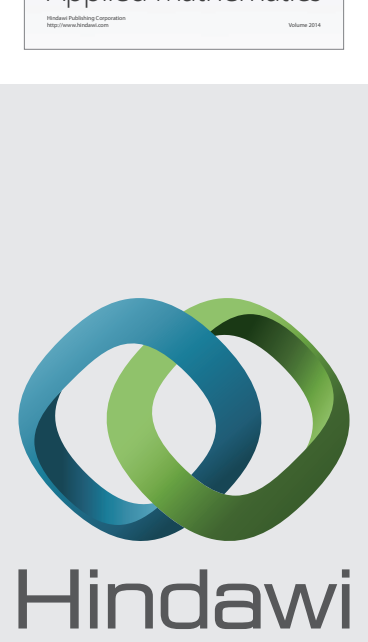

Submit your manuscripts at http://www.hindawi.com
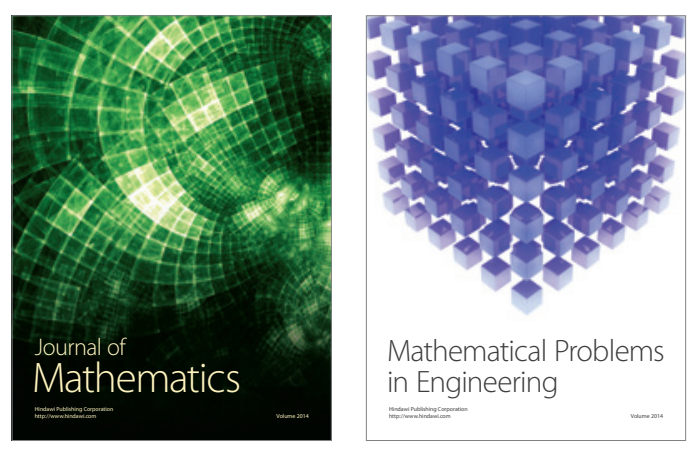

Mathematical Problems in Engineering
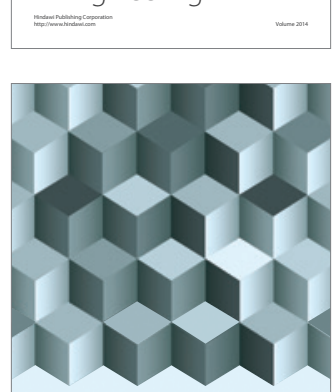

Journal of

Function Spaces
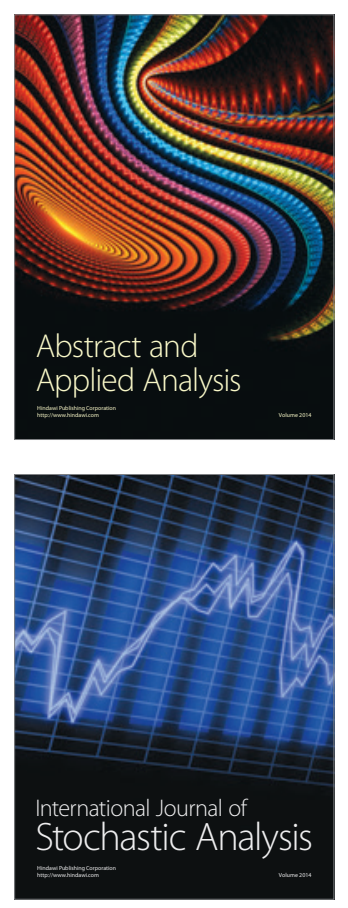

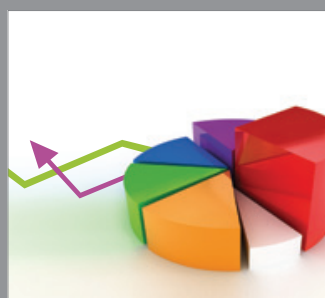

ournal of

Probability and Statistics

Promensencen
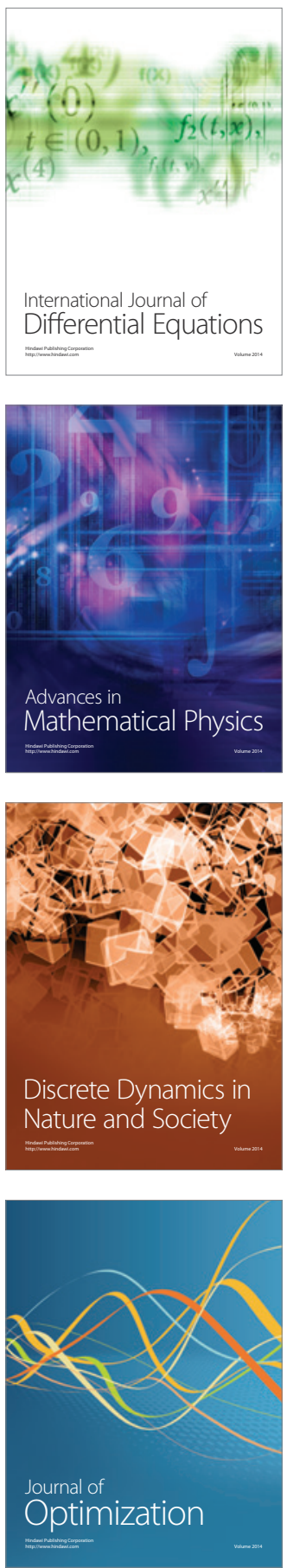